\title{
Existence and uniqueness of traveling waves for fully overdamped Frenkel-Kontorova models
}

\author{
M. Al Haj, N. Forcadel, R. Monneau ${ }^{1}$
}

March 19, 2013

\begin{abstract}
In this article, we study the existence and the uniqueness of traveling waves for a discrete reaction-diffusion equation with bistable non-linearity, namely a generalization of the fully overdamped Frenkel-Kontorova model. This model consists in a system of ODE's which describes the dynamics of crystal defects in a lattice solids. Under very poor assumptions, we prove the existence of a traveling wave solution and the uniqueness of the velocity of propagation of this traveling wave. The question of the uniqueness of the profile is also studied by proving Strong Maximum Principle or some weak asymptotics on the profile at infinity.
\end{abstract}

Keywords: Frenkel-Kontorova models, traveling waves, viscosity solutions, comparison principle.

\section{Introduction}

In this work, we are interested in the fully overdamped Frenkel-Kontorova (FK) model which describes the dynamics of crystal defects in a lattice (see for instance the book of Braun and Kivshar [8] for an introduction to this model). This model (and its generalization) is a discrete reaction-diffusion equation with "bistable" non-linearity. For this model, we show the existence and the uniqueness of traveling waves.

\subsection{Setting of the problem}

We first give an example of the simplest fully overdamped Frenkel Kontorova model, and then we provide a general framework for which we will establish our results.

(i) The simplest Frenkel-Kontorova model

The simplest fully overdamped FK model is a chain of atoms, where the position $X_{i}(t) \in \mathbb{R}$ at the time $t$ of the particle $i \in \mathbb{Z}$ solves

$$
\frac{d X_{i}}{d t}=X_{i+1}+X_{i-1}-2 X_{i}-\sin \left(2 \pi\left(X_{i}-L\right)\right)-\sin (2 \pi L)
$$

\footnotetext{
${ }^{1}$ Université Paris-Est, CERMICS, Ecole des Ponts ParisTech, 6-8 avenue Blaise Pascal, 77455 Marne-laVallée Cedex 2, France

${ }^{2}$ Université Paris-Dauphine, CEREMADE, UMR CNRS 7534, place de Lattre de Tassigny, 75775 Paris cedex 16
} 
where $\frac{d X_{i}}{d t}$ is the velocity of the $i$ th particle, $-\sin (2 \pi L)$ is a constant driving force which will cause the movement of the chain of atoms and $\sin \left(2 \pi X_{i}\right)$ denotes the force created by a periodic potential reflecting the periodicity of the crystal, whose period is assumed to be 1. Set, for simplicity,

$$
f_{L}(x):=-\sin (2 \pi(x-L))-\sin (2 \pi L)
$$

We look for particular traveling wave solutions of (1.1), namely solutions of the form

$$
X_{i}(t)=\phi(i+c t)
$$

with

$$
\left\{\begin{array}{l}
\phi^{\prime} \geq 0 \\
\phi(+\infty)-\phi(-\infty)=1
\end{array}\right.
$$

Here $c$ is the velocity of propagation of the traveling wave $\phi$, and (1.4) reflects the existence of a defect of one lattice space, called dislocation. Moreover, expression (1.3) means that the defect moves with velocity $c$ under the driving force $L$. In addition, $\phi$ is a phase transition between $\phi(-\infty)$ and $\phi(+\infty)$ which are two "stable" equilibriums of the crystal.

Clearly, if we plug (1.3) in (1.1), the profile $\phi$ and the velocity $c$ have to satisfy

$$
c \phi^{\prime}(z)=\phi(z+1)+\phi(z-1)-2 \phi(z)+f_{L}(\phi(z)),
$$

with $z=i+c t$ and $f_{L}$ defined in (1.2).

Due to the equivalence (for $c \neq 0$ ) between solutions of (1.1) and (1.5), from now on, we will focus on equation (1.5).

Theorem 1.1 (Existence and uniqueness of traveling waves for (FK) model) There exists a unique real $c$ and a function $\phi: \mathbb{R} \rightarrow \mathbb{R}$ solution of

$$
\left\{\begin{array}{l}
c \phi^{\prime}(z)=\phi(z+1)+\phi(z-1)-2 \phi(z)+f_{L}(\phi(z)) \text { on } \mathbb{R} \\
\phi \text { is non-decreasing over } \mathbb{R} \\
\phi(-\infty)=0 \quad \text { and } \phi(+\infty)=1,
\end{array}\right.
$$

in classical sense if $c \neq 0$ and almost every where if $c=0$. Moreover, if $c \neq 0$, then the profile $\phi$ is unique (up to space translation) and $\phi^{\prime}>0$ on $\mathbb{R}$.

This theorem has be proved in several works (see for instance, the pioneering works [25] and [18], and [22] in full generality).

(ii) A simple example not covered by the literature

Define the function $G$ as

$$
G\left(X_{i-1}, X_{i}, X_{i+1}\right):=\max \left(\frac{1}{2}, X_{i-1}\right)+\min \left(\frac{1}{2}, X_{i+1}\right)-X_{i}-\frac{1}{2}+f_{L}\left(X_{i}\right),
$$

where $f_{L}$ defined in (1.2), then consider the following system

$$
\dot{X}_{i}=G\left(X_{i-1}, X_{i}, X_{i+1}\right) \quad \text { for } \quad i \in \mathbb{Z}
$$


Theorem 1.2 (Existence and uniqueness of traveling waves for example (1.7)) For any $L \in\left(\frac{-1}{4}, \frac{1}{4}\right) \backslash\{0\}$, the results of Theorem 1.1 hold true for system (1.6) replaced by the following system

$$
\left\{\begin{array}{l}
c \phi^{\prime}(z)=G(\phi(z-1), \phi(z), \phi(z+1)) \quad \text { on } \mathbb{R} \\
\phi \text { is non-decreasing over } \mathbb{R} \\
\phi(-\infty)=0 \text { and } \phi(+\infty)=1 .
\end{array}\right.
$$

Up to our knowledge, this result is new. Notice that this result is for instance not included in Mallet-Paret's work [22], since $G$ does not satisfy $\frac{\partial G}{\partial X_{i-1}}>0$ and $\frac{\partial G}{\partial X_{i+1}}>0$. Such a condition is important in [22] to construct the traveling waves using deformation (continuation) method.

\section{(iii) General framework}

We now consider a generalization of equation (1.5). To this end, we introduce a real function (whose properties to be specified later in Subsection 1.2):

$$
F:[0,1]^{N+1} \rightarrow \mathbb{R}
$$

We then consider the following equation

$$
c \phi^{\prime}(z)=F\left(\phi\left(z+r_{0}\right), \phi\left(z+r_{1}\right), \ldots, \phi\left(z+r_{N}\right)\right),
$$

where $N \geq 0$ and $r_{i} \in \mathbb{R}$ for $i=0, \ldots, N$. We also normalize the limits of the profile at infinity as follows:

$$
\phi(-\infty)=0, \quad \phi(+\infty)=1 .
$$

Note that, for $N=2$ and $F=F_{0}\left(X_{0}, X_{1}, X_{2}\right)=X_{2}+X_{1}-2 X_{0}+f_{L}\left(X_{0}\right)$, equation (1.5) is a particular case of (1.11). Moreover, $F_{0}$ is compatible with (1.12).

Assume, without loss of generality, for the whole work that:

$$
r_{0}=0 \quad \text { and } \quad r_{i} \neq r_{j} \text { if } i \neq j .
$$

\subsection{Main results}

In order to present our results, we have to introduce some assumptions on $F$ defined in (1.10). Note that, for later use, we split these assumptions into assumptions $(A)$ and $(B)$.

\section{Assumption (A):}

Regularity: $F$ is globally Lipschitz continuous over $[0,1]^{N+1}$.

Monotonicity: $F\left(X_{0}, \ldots, X_{N}\right)$ is non-decreasing w.r.t. each $X_{i}$ for $i \neq 0$. 
We set $f(v)=F(v, \ldots, v)$.

\section{Assumption (B):}

Instability: $f(0)=0=f(1)$ and there exists $b \in(0,1)$ such that $f(b)=0$, $f_{\left.\right|_{(0, b)}}<0, f_{\left.\right|_{(b, 1)}}>0$ and $f^{\prime}(b)>0$.

Smoothness: $F$ is $C^{1}$ in a neighborhood of $\{b\}^{N+1}$.

\section{Remark 1.3}

1. The point $b$ is supposed to be unstable and this is the meaning of the condition $f^{\prime}(b)>0$.

2. Notice that the instability part of assumption $(B)$ means in particular that $f$ is of "Bistable" shape (see [22]).

\section{Theorem 1.4 (Existence of a traveling wave)}

Under assumptions $(A),(B)$, there exist a real $c \in \mathbb{R}$ and a function $\phi: \mathbb{R} \rightarrow \mathbb{R}$ that solves

$$
\left\{\begin{array}{l}
c \phi^{\prime}(z)=F\left(\phi\left(z+r_{0}\right), \phi\left(z+r_{1}\right), \ldots, \phi\left(z+r_{N}\right)\right) \text { on } \mathbb{R} \\
\phi \text { is non-decreasing over } \mathbb{R} \\
\phi(-\infty)=0 \quad \text { and } \phi(+\infty)=1
\end{array}\right.
$$

in the classical sense if $c \neq 0$ and almost every where if $c=0$.

Our method to construct a solution relies on the construction of a hull function for an associated homogenization problem (see the work of Forcadel, Imbert, Monneau [23]). In order to prove the uniqueness of the traveling wave, we need the following additional assumptions:

Assumption (C): Inverse monotonicity close to $\{0\}^{N+1}$ and $E=\{1\}^{N+1}$ There exists $\beta_{0}>0$ such that for $a>0$, we have

$$
\left\{\begin{array}{lll}
F(X+(a, \ldots, a))<F(X) & \text { for all } & X, X+(a, \ldots, a) \in\left[0, \beta_{0}\right]^{N+1} \\
F(X+(a, \ldots, a))<F(X) & \text { for all } & X, X+(a, \ldots, a) \in\left[1-\beta_{0}, 1\right]^{N+1}
\end{array}\right.
$$

This condition is important to get the comparison principle (see Theorem 4.1).

Assumption (D+):

i) All the $r_{i}$ 's "Shifts" have the same sign: Assume that $r_{i} \leq 0$ for all $i \in\{0, \ldots, N\}$.

ii) Strict monotonicity: $F$ is increasing in $X_{i^{+}}$with $r_{i^{+}}>0$.

Assumption (D-):

i) All the $r_{i}$ 's "Shifts" have the same sign: Assume that $r_{i} \geq 0$ for all $i \in\{0, \ldots, N\}$.

ii) Strict monotonicity: $F$ is increasing in $X_{i^{-}}$with $r_{i^{-}}<0$.

Assumption (E+):

i) Strict monotonicity close to 0 : Assume that $\frac{\partial F}{\partial X_{i^{+}}}(0)>0$ with $r_{i^{+}}>0$. 
ii) Smoothness close to $\{0\}^{N+1}$ :

There exists $\nabla F(0)$, with $f^{\prime}(0)<0$, and there exists $\alpha \in(0,1)$ and $C_{0}>0$ such that for all $X \in[0,1]^{N+1}$

$$
|F(X)-F(0)-X . \nabla F(0)| \leq C_{0}|X|^{1+\alpha} .
$$

Assumption (E-):

i) Strict monotonicity close to 1 : Assume, for $E=(1, \ldots, 1) \in \mathbb{R}^{N+1}$, that $\frac{\partial F}{\partial X_{i^{-}}}(E)>0$ with $r_{i^{-}}<0$.

ii) Smoothness close to $\{1\}^{N+1}$ :

There exists $\nabla F(E)$ with $f^{\prime}(1)<0$ and there exists $\alpha \in(0,1)$ and $C_{0}>0$ such that for all $X \in[0,1]^{N+1}$

$$
|F(X)-F(E)-(X-E) \cdot \nabla F(E)| \leq C_{0}|X-E|^{1+\alpha},
$$

with $E=(1, \ldots, 1) \in \mathbb{R}^{N+1}$.

Theorem 1.5 (Uniqueness of the velocity and of the profile)

Assume $(A)$ and let $(c, \phi)$ be a solution of

$$
\left\{\begin{array}{l}
c \phi^{\prime}(z)=F\left(\phi\left(z+r_{0}\right), \phi\left(z+r_{1}\right), \ldots, \phi\left(z+r_{N}\right)\right) \text { on } \mathbb{R} \\
\phi(-\infty)=0 \text { and } \phi(+\infty)=1 .
\end{array}\right.
$$

(a) Uniqueness of the velocity: Under the additional assumption $(C)$, the velocity $c$ is unique.

(b) Uniqueness of the profile $\phi$ : If $c \neq 0$, then under the additional assumptions $(C)$ and $(D+) i)$ or $i i)$ or $(E+)$ if $c>0($ resp. $(D-) i)$ or ii) or $(E-)$ if $c<0)$, the profile $\phi$ is unique (up to space translation) and $\phi^{\prime}>0$ on $\mathbb{R}$.

\section{Remark 1.6 (Interpreting the assumptions)}

(1) If $\mathbf{c}>\mathbf{0}$ : Assumptions $(D+) i)$, ii) and $(E+)$ are respectively important to prove a Strong Maximum Principle (cf Lemma 6.2 and 6.4) and the asymptotics of the profile near $-\infty$ (cf Lemma 6.6) that we use to prove the uniqueness of the profile of a solution.

(2) If $\mathbf{c}<\mathbf{0}$ : Under $(D-) i)$, ii) and $(E-)$, we respectively get the same results as for $c>0$, however, the asymptotics are proven near $+\infty$ in this case.

Remark also that Theorems 1.1 and 1.2 are particular cases of Theorem 1.4 and Theorem 1.5. Indeed, existence of the solution in Theorem 1.2 follows from Theorem 1.4 and the fact that $b \neq \frac{1}{2}$ in assumption $(B)$, when $L \in\left(-\frac{1}{4}, \frac{1}{4}\right) \backslash\{0\}$. Uniqueness of the profile in Theorem 1.2 follows from Theorem 1.5 (b), and the fact that the function $G$ defined in (1.7) verifies assumptions $(E \pm)$.

For the whole paper, we define

$$
r^{*}=\max _{i=0, \ldots, N}\left|r_{i}\right|
$$

and we set, as a notation, for a general function $h$ :

$$
F\left(\left(h\left(y+r_{i}\right)\right)_{i=0, \ldots, N}\right):=F\left(h\left(y+r_{0}\right), h\left(y+r_{1}\right), \ldots, h\left(y+r_{N}\right)\right) .
$$




\subsection{Brief review of the literature}

The study of traveling waves in reaction-diffusion equations has been introduced in pioneering works of Fisher [16] and Kolmogorov, Petrovsky and Piskunov [20]. Existence of traveling waves solutions has been for instance obtained in $[2,7,19,15]$. More generally, there is a huge literature about existence, uniqueness and stability of traveling waves with various non linearities with applications in particular in biology and combustion and we refer for instance to the references cited in $[6,10]$. There are also several works on discrete or nonlocal versions of reactions-diffusion equations (see for instance $[5,9,11,13,14,17,24,26]$ and $[10,22]$ and the references cited therein).

As explained above, in the case of bistable non-linearity $f$, the existence and uniqueness of traveling waves are well known for the model equation

$$
u_{t}=u_{x x}+f(u)
$$

Starting from equation (1.16), and using a continuation method, Bates et al. [4] proved in particular the existence of traveling waves for the convolution model

$$
u_{t}=J * u-u+f(u)
$$

where $J$ is a kernel.

In [22], Mallet-Paret (see also Carpio et al. [9] for semi-linear case) used also a global continuation method (i.e. a homotopy method) to get existence of traveling waves for bistable non-linearities and information about the uniqueness and the dependence of solutions on parameters. This continuation argument was applied to connect the discrete dynamical system that he studied and a PDE model (similar to (1.16)) for which the existence and uniqueness are known. He proved the continuation between the solutions of the two systems using a general Fredholm alternative method [21] for the linearized traveling waves equations.

Traveling waves were also studied by Chow et al. [12] for lattice dynamical systems (lattice ODE's) and for coupled maps lattices (CML's) that arise as time-discretizations of lattice ODE's. Using a geometric approach, the authors studied the stability of traveling waves for lattice ODE's and proved existence of traveling waves of their time discretized CML's. More precisely, they constructed a local coordinate system in a tubular neighborhood of the traveling wave solution in the phase space of their system. Such an approach is used to transform lattice ODE's into a nonautonomous time-periodic ODE and traveling waves to periodic solutions of this ODE. In addition, they gain from this transformation the possibility to use the standard tools of dynamical systems and to see traveling waves of CML as certain orbits for a circle diffeomorphism whose rotational number is equal to the wave speed.

Zinner [25] proved the existence of traveling waves for the discrete Nagumo equation

$$
\dot{x}_{i}=d\left(x_{i+1}-2 x_{i}+x_{i-1}\right)+f\left(x_{i}\right) \quad i \in \mathbb{Z} .
$$

The construction is done introducing first a simplified problem (using a projection to 0 or 1 for $|i| \geq N)$ for which the existence is attained by Brouwer's fixed point theorem. Hankerson and Zinner [18] also proved existence of traveling waves (for an equation more general than (1.18)) obtained as the long time limit of the solution with Heaviside initial data, using an interesting lap number argument.

In [10], Chen, Guo and Wu constructed traveling waves for a lattice ODE's with bistable non-linearity. They rephrase the solution $\phi$ of (1.11) as a fixed point of an integral formulation. First, they considered a simplified problem (using a projection on 0 or 1 for large 
indices $|i| \geq N)$ and they show, for any $c \neq 0$, the existence of a solution $\phi^{N, c}$ using the monotone iteration method. Finally, they recover the existence of a solution in the limit $N \rightarrow+\infty$ for a suitable choice $c=c(N)$ converging to a limit velocity.

In this paper, we introduce a completely new method at least to prove the existence of traveling waves. In our approach, the existence of traveling waves relies on the construction of hull functions of slope $p$ (like correctors) for an associated homogenization problem. Passing to the limit $p \rightarrow 0$, one important difficulty is to identify a traveling wave joining two stable states. In particular, we have avoided this traveling wave to degenerate to the intermediate unstable state. The uniqueness of the profile is proved using either strong maximum principle or weak asymptotics of the profile. Notice that, using weak asymptotics (in comparison with those of Mallet-Paret [22]) allow us to have weaker assumptions.

We also mention that our method is still effective in higher dimensional problems. Consider, for instance, the model

$$
\frac{d}{d t} X_{I}(t)=f\left(X_{I}\right)+\sum_{|J|=1}\left(X_{I+J}-X_{I}\right)
$$

that describes the interaction of an atom $I \in \mathbb{Z}^{n}$ with its nearest neighbors $\left(X_{I} \in \mathbb{R}\right.$ denotes the position of atom $I)$. We can look for traveling waves $X_{I}(t)=\phi(c t+\nu \cdot I)$ that propagates in a direction $\nu \in \mathbb{R}^{n}$ with $|\nu|=1$. That is for $z=c t+\nu \cdot I$, we look for $\phi$ solution of

$$
c \phi^{\prime}(z)=f(\phi(z))+\sum_{|J|=1}(\phi(z+\nu \cdot J)-\phi(z)),
$$

where $f$ denotes a bistable non-linearity. Setting $r_{j}:=\nu \cdot J$, we recover an equation of type (1.11) for $N=2 n$. Therefore, the results of higher dimensional problems follow from our one dimensional results (Theorems 1.4 and 1.5) as far as they hold for general shifts $r_{j}$ 's.

We get the existence of solutions under very poor assumptions in comparison with similar results in previous works. Our framework is very flexible, and does not require a setting in any particular functional space. We also think that our method opens new perspectives and could be used to study many models: for example, fully overdamped FK models with time dependent non-linearities, accelerated FK models, FK with multi-particles.

\subsection{Organization of the paper}

In Section 2, we introduce an extension of $F$ onto $\mathbb{R}^{N+1}$ and we recall, for the extension function, the notion of viscosity solutions, the existence of hull functions for our model and we prove some results about monotone functions. We prove Theorem 1.4 (for the extended function) in Section 3. In Section 4, we prove the uniqueness of the velocity of a profile (Theorem 1.5 part $(a)=$ Proposition 4.5$)$ and a comparison principle result on the half-line. Section 5 is devoted to the asymptotics of a profile near $\pm \infty$ (Proposition 5.1). In Section 6 , we prove the uniqueness of the profile (Theorem 1.5 part $(b)$ ). Finally, we prove in the Appendices A and B the extension result, namely Lemma 2.1 and some results about monotone function, namely Lemmas 2.10 and 2.11 respectively.

\section{Preliminary results}

This section is divided into four subsections. In the first subsection, we extend the function $F$ onto $\mathbb{R}^{N+1}$. In the second subsection, we recall the definition of a viscosity solution. We 
apply a result of existence of hull functions associated to the homogenization of our problem with the extended $F$ in the third subsection. We dedicate the fourth subsection for some results about monotone functions that we will use in Section 3.

\subsection{Extension of $F$}

The proof of existence of traveling waves is based on the construction of hull functions (like correctors) associated to a homogenization problem (see [23]). To this end, we first need to extend the function $F$ in $\tilde{F}$ defined over $\mathbb{R}^{N+1}$ and satisfying the following assumption:

\section{Assumption $(\tilde{\boldsymbol{A}})$ :}

Regularity: $\tilde{F}$ is globally Lipschitz continuous over $\mathbb{R}^{N+1}$.

Periodicity: $\tilde{F}\left(X_{0}+1, \ldots, X_{N}+1\right)=\tilde{F}\left(X_{0}, \ldots, X_{N}\right)$ for every $X=\left(X_{0}, \ldots, X_{N}\right) \in \mathbb{R}^{N+1}$.

Monotonicity: $\tilde{F}\left(X_{0}, \ldots, X_{N}\right)$ is non-decreasing w.r.t. each $X_{i}$ for $i \neq 0$.

The extension result is the following:

\section{Lemma 2.1 (Extension of $F$ )}

Given a function $F$ defined over $Q=[0,1]^{N+1}$ satisfying $(A)$ and $F(1, \ldots, 1)=F(0, \ldots, 0)$, there exists an extension $\tilde{F}$ defined over $\mathbb{R}^{N+1}$ such that

$$
\tilde{F}_{\left.\right|_{Q}}=F \quad \text { and } \quad \tilde{F} \text { satisfies }(\tilde{A}) \text {. }
$$

The proof of this lemma is postponed in Appendix A.

Remark 2.2 We notice that, if $\phi$ is a traveling wave constructed for (1.13) with $F$ replaced by $\tilde{F}$, then $\phi$ is a traveling wave of (1.13). This is a direct consequence of Lemma 2.1 and the fact that

$$
\left\{\begin{array}{l}
\phi \text { is non-decreasing on } \mathbb{R} \\
\phi(-\infty)=0 \text { and } \phi(+\infty)=1 .
\end{array}\right.
$$

By convention, we will say that $\tilde{F}$ satisfies $(B)(\operatorname{resp} .(C),(D)$ or $(E))$ if and only if $F=\tilde{F}_{\left.\right|_{Q}}$ satisfies $(B)$ (resp. $(C),(D)$ or $(E))$.

We now give a result corresponding to Theorem 1.4 for $\tilde{F}$, whose proof is given in Section 3 .

Proposition 2.3 (Result corresponding to Theorem 1.4 for $\tilde{F}$ ) Assume that $\tilde{F}$ satisfies $(\tilde{A}),(B)$. Then there exist a real $c$ and a function $\phi$ solution of

$$
\left\{\begin{array}{l}
c \phi^{\prime}(z)=\tilde{F}\left(\left(\phi\left(z+r_{i}\right)\right)_{i=0, \ldots, N}\right) \text { on } \mathbb{R} \\
\phi \text { is non-decreasing on } \mathbb{R} \\
\phi(-\infty)=0 \quad \text { and } \quad \phi(+\infty)=1,
\end{array}\right.
$$

in the classical sense if $c \neq 0$ and almost everywhere if $c=0$.

For simplicity, in the rest of this section and in Section 3, we call $\tilde{F}$ as $F$.

\section{Proof of Theorem $\mathbf{1 . 4}$}

The proof of Theorem 1.4 is a straightforward consequence of Remark 2.2 and Proposition 2.3 . 


\subsection{Viscosity solution}

In the whole paper, we will use the notion of viscosity solution that we introduce in this subsection. To this end, we recall that the upper and the lower semi-continuous envelopes, $u^{*}$ and $u_{*}$, of a locally bounded function $u$ are defined as

$$
u^{*}(y)=\limsup _{x \rightarrow y} u(x) \text { and } u_{*}(y)=\liminf _{x \rightarrow y} u(x) .
$$

\section{Definition 2.4 (Viscosity solution)}

Let $u: \mathbb{R} \rightarrow \mathbb{R}$ be a locally bounded function, $c \in \mathbb{R}$ and $F$ defined on $\mathbb{R}^{N+1}$.

- The function $u$ is a sub-solution (resp. a super-solution) of

$$
c u^{\prime}(x)=F\left(\left(u\left(x+r_{i}\right)\right)_{i=0, \ldots, N}\right) \quad \text { on } \quad \mathbb{R},
$$

if $u$ is upper semi-continuous (resp. lower semi-continuous) and if for all test function $\psi \in C^{1}(\mathbb{R})$ such that $u-\psi$ attains a local maximum (resp. a local minimum) at $x^{*}$, we have

$$
c \psi^{\prime}\left(x^{*}\right) \leq F\left(\left(u\left(x^{*}+r_{i}\right)\right)_{i=0, \ldots, N}\right) \quad\left(\operatorname{resp} . c \psi^{\prime}\left(x^{*}\right) \geq F\left(\left(u\left(x^{*}+r_{i}\right)\right)_{i=0, \ldots, N}\right)\right) .
$$

- A function $u$ is a viscosity solution of (2.21) if $u^{*}$ is a sub-solution and $u_{*}$ is a supersolution.

We also recall the stability result for viscosity solutions (see [3, Theorem 4.1]).

\section{Proposition 2.5 (Stability of viscosity solutions)}

Consider a function $F$ defined on $\mathbb{R}^{N+1}$ and satisfying $(\tilde{A})$. Assume that $\left(u_{\varepsilon}\right)_{\varepsilon}$ is a sequence of sub-solutions (resp. super-solutions) of (2.21). Suppose that the functions $\left(u_{\varepsilon}\right)_{\varepsilon}$ are uniformly locally bounded on $\mathbb{R}$ and let

$$
\bar{u}(x)=\limsup _{\varepsilon \rightarrow 0} u_{\varepsilon}(x):=\limsup _{(\varepsilon, y) \rightarrow(0, x)} u_{\varepsilon}(y) \quad \text { and } \quad \underline{u}(x)=\liminf _{\varepsilon \rightarrow 0} u_{\varepsilon}(x):=\liminf _{(\varepsilon, y) \rightarrow(0, x)} u_{\varepsilon}(y),
$$

be the relaxed upper and lower semi-limits. If $\bar{u}$ (resp. $\underline{u}$ ) is finite, then $\bar{u}$ is a sub-solution (resp. $\underline{u}$ is a super-solution) of (2.21).

\subsection{On the hull function}

In this subsection, we first adapt the result of existence of a hull function associated to the homogenization of our problem, then we make the link between the existence of a hull function and the existence of the traveling wave.

Lemma 2.6 (Existence of a hull function ([23, Theorem 1.5]))

Let $F$ be a given function satisfying assumption $(\tilde{A})$ and $p>0$. There exists a unique $\lambda_{p}$ such that there exists a locally bounded function $h_{p}: \mathbb{R} \rightarrow \mathbb{R}$ satisfying (in the viscosity sense):

$$
\left\{\begin{array}{l}
\lambda_{p} h_{p}^{\prime}=F\left(\left(h_{p}\left(y+p r_{i}\right)\right)_{i=0, \ldots, N}\right) \quad \text { on } \quad \mathbb{R} \\
h_{p}(y+1)=h_{p}(y)+1 \\
h_{p}^{\prime}(y) \geq 0 \\
\left|h_{p}\left(y+y^{\prime}\right)-h_{p}(y)-y^{\prime}\right| \leq 1 \text { for all } y^{\prime} \in \mathbb{R}
\end{array}\right.
$$


Such a function $h_{p}$ is called a hull function. Moreover, there exists a constant $K>0$, independent on $p$, such that

$$
\left|\lambda_{p}\right| \leq K(1+p)
$$

Notice that Lemma 2.6 is proven in [23] only for $r_{i} \in \mathbb{Z}$. However, the proof for the generalization $r_{i} \in \mathbb{R}$ is still valid (it is exactly the same).

After this recall, and using the hull function $h_{p}$, we define the function $\phi_{p}$ as:

$$
\phi_{p}(x):=h_{p}(p x)
$$

Moreover we set, as a velocity, the ratio

$$
c_{p}:=\frac{\lambda_{p}}{p}
$$

Remark 2.7 It is possible that $c_{p}=0$ for all $p>0$. Our proof of existence of traveling wave is done for the general case. However, we state throw out the proof the different situations for the velocity.

Notice that the above $\phi_{p}$ satisfies the following lemma:

Lemma 2.8 (Properties of $\phi_{p}$ )

Let $p>0$ and assume $(\tilde{A})$. Then the function $\phi_{p}$ defined in (2.23) satisfies in the viscosity sense:

$$
\left\{\begin{array}{l}
c_{p} \phi_{p}^{\prime}=F\left(\left(\phi_{p}\left(z+r_{i}\right)\right)_{i=0, \ldots, N}\right) \quad \text { on } \quad \mathbb{R} \\
\phi_{p}^{\prime} \geq 0 \\
\phi_{p}\left(z+\frac{1}{p}\right)=\phi_{p}(z)+1
\end{array}\right.
$$

Moreover, if $c_{p} \neq 0$ then there exists $M>0$ independent on $p$ such that

$$
\left|\phi_{p}^{\prime}\right| \leq \frac{M}{\left|c_{p}\right|}
$$

for $0<p \leq \frac{1}{r^{*}}$, with $r^{*}$ given in (1.15).

\section{Proof of Lemma 2.8.}

Let $h_{p}$ be a viscosity solution given by Lemma 2.6. Then we get $(2.25)$ by the change of variables (2.23)-(2.24). We now show (2.26). We choose $p>0$ such that

$$
\frac{1}{p} \geq r^{*}
$$

Since $\phi_{p}$ is non-decreasing, then we have

$$
\left\{\begin{array}{l}
\left|\phi_{p}\left(x+r_{i}\right)-\phi_{p}(x)\right| \leq\left|\phi_{p}\left(x+\frac{1}{p}\right)-\phi_{p}(x)\right|=1 \quad \text { if } \quad r_{i} \geq 0 \\
\left|\phi_{p}\left(x+r_{i}\right)-\phi_{p}(x)\right| \leq\left|\phi_{p}\left(x-\frac{1}{p}\right)-\phi_{p}(x)\right|=1 \quad \text { if } \quad r_{i} \leq 0
\end{array}\right.
$$


Moreover, since $F \in \operatorname{Lip}\left(\mathbb{R}^{N+1}\right)$, then

$$
\left|F\left(\left(\phi_{p}\left(x+r_{i}\right)_{i=0, \ldots, N}\right)\right)-F\left(\left(\phi_{p}(x)\right)_{i=0, \ldots, N}\right)\right| \leq L\left|\begin{array}{c}
1 \\
\vdots \\
1
\end{array}\right|=: L^{1}
$$

where $L$ is the Lipschitz constant of $F$. On the other hand, $f$ is bounded (because $f$ is Lipschitz and periodic) and $F\left(\left(\phi_{p}(x)\right)_{i=0, \ldots, N}\right)=f\left(\phi_{p}(x)\right)$, thus

$$
\left|F\left(\left(\phi_{p}\left(x+r_{i}\right)_{i=0, \ldots, N}\right)\right)\right| \leq L^{1}+|f|_{L^{\infty}}=: M .
$$

This implies that

$$
\left|c_{p} \phi_{p}^{\prime}\right| \leq M
$$

in the viscosity sense. If in addition $c_{p} \neq 0$, then we get the Lipschitz bound

$$
\left|\phi_{p}^{\prime}\right| \leq \frac{M}{\left|c_{p}\right|} .
$$

\subsection{Useful results about monotone functions}

In this subsection, we recall miscellaneous results about monotone functions that we will use later in Section 3 for the proof of Proposition 2.3. We state Helly's Lemma on the one hand, and the equivalence between viscosity and almost everywhere solution on the other hand.

Lemma 2.9 (Helly's Lemma, (see [1], Section 3.3, page 70))

Let $\left(g_{n}\right)_{n \in \mathbb{N}}$ be a sequence of non-decreasing functions on $[a, b]$ verifying $\left|g_{n}\right| \leq M$ uniformly in $n$. Then there exists a subsequence $\left(g_{n_{j}}\right)_{j \in \mathbb{N}}$ such that

$$
g_{n_{j}} \rightarrow g \text { a.e. on }[a, b]
$$

with $g$ non-decreasing and $|g| \leq M$.

\section{Lemma 2.10 (Complement of Helly's Lemma)}

Let $\left(g_{n}\right)_{n \in \mathbb{N}}$ be a sequence of non-decreasing functions on a bounded interval $I$ and suppose that

$$
g_{n} \rightarrow g \text { a.e. on } I \text {. }
$$

If $g$ is constant on $\stackrel{\circ}{I}$, then for every closed subset interval $I^{\prime} \subset \stackrel{\circ}{I}$,

$$
g_{n} \rightarrow g \text { uniformly on } I^{\prime} \text {. }
$$

The proof of Lemma 2.10 is done in Appendix B.

We introduce now a lemma that shows the equivalence between viscosity and almost everywhere solutions under the monotonicity of the solution.

Lemma 2.11 (Equivalence between viscosity and a.e. solutions)

Let $F$ satisfying assumption $(\tilde{A})$. Let $\phi: \mathbb{R} \rightarrow \mathbb{R}$ be a non-decreasing function. Then $\phi$ is a viscosity solution of

$$
0=F\left(\left(\phi\left(x+r_{i}\right)\right)_{i=0, \ldots, N}\right) \quad \text { on } \quad \mathbb{R}
$$

if and only if $\phi$ is an almost everywhere solution of the same equation.

The proof of Lemma 2.11 is also delayed in Appendix B. 


\section{Construction of a traveling wave: proof of Proposi- tion 2.3}

This section is devoted to the proof of existence of a traveling wave for system (2.20). We control both the velocity of propagation and the finite difference of a solution in the first subsection. Then we prove Proposition 2.3 in the second subsection.

\subsection{Preliminary results}

We have

\section{Lemma 3.1 (Velocity $c_{p}$ is bounded)}

Under the assumption $(\tilde{A}),(B)$, let $c_{p}$ be the velocity given by (2.24). Then there exists $M_{1}>0$ such that

$$
\left|c_{p}\right| \leq M_{1}
$$

for $0<p \leq \frac{1}{r^{*}}$, with $r^{*}$ given in (1.15).

Proof of Lemma 3.1.

Consider the function $\phi_{p}$ given by (2.23) which satisfies (2.25). Let $c_{p}$ be the associated velocity given by (2.24) and assume by contradiction that when $p \rightarrow p_{0} \in\left[0, \frac{1}{r^{*}}\right]$

$$
\lim _{p \rightarrow p_{0}} c_{p}=+\infty
$$

(the case $c_{p} \rightarrow-\infty$ being similar). Let $\bar{\phi}_{p}(x):=\phi_{p}\left(c_{p} x\right)$ solution of

$$
\bar{\phi}_{p}^{\prime}(x)=F\left(\left(\bar{\phi}_{p}\left(x+\frac{r_{i}}{c_{p}}\right)\right)_{i=0, \ldots, N}\right) .
$$

Since $\bar{\phi}_{p}$ is invariant w.r.t. space translations, we may assume that

$$
\bar{\phi}_{p}(0)=b-\varepsilon
$$

for some $\varepsilon>0$ small enough. Moreover, by (2.26) we have

$$
\left|\bar{\phi}_{p}^{\prime}\right|=\left|c_{p} \phi_{p}^{\prime}\right| \leq M
$$

for some $M>0$ independent on $p$. Thus using Ascoli's Theorem and the diagonal extraction argument, $\bar{\phi}_{p}$ converges as $p \rightarrow p_{0}$ (up to a subsequence) to some $\bar{\phi}$ locally uniformly on $\mathbb{R}$, and $\bar{\phi}$ satisfies classically

$$
\begin{aligned}
\bar{\phi}^{\prime}(x) & =F\left((\bar{\phi}(x))_{i=0, \ldots, N}\right) \\
& =f(\bar{\phi}(x))
\end{aligned}
$$

and $\bar{\phi}(0)=b-\varepsilon$. But $\bar{\phi}_{p}^{\prime} \geq 0$ (because (3.28) implies trivially that $c_{p} \geq 0$ ), thus $\bar{\phi}^{\prime} \geq 0$. Hence $f(\bar{\phi}(x)) \geq 0$ for all $x$, in particular $f(\bar{\phi}(0))=f(b-\varepsilon)$, a contradiction since $f(b-\varepsilon)<0$ (see assumption $(B))$.

Next, we introduce an important proposition on the control of the finite difference that will be used in the proof of existence of a traveling wave. 


\section{Proposition 3.2 (Control on the finite difference)}

Assume that $F$ satisfies $(\tilde{A})$ and let $a>r^{*}$ with $r^{*}$ given by (1.15) and $M_{0}>0$. For every $\varepsilon>0$, there exists $\delta>0$ such that for all function $\phi$ (viscosity) solution of

$$
\left\{\begin{array}{l}
c \phi^{\prime}(x)=F\left(\left(\phi\left(x+r_{i}\right)\right)_{i=0, \ldots, N}\right) \quad \text { on } \quad \mathbb{R} \\
\phi^{\prime} \geq 0 \\
\phi(x+1) \leq \phi(x)+1 \\
|c| \leq M_{0} \\
\left|c \phi^{\prime}\right| \leq M_{0},
\end{array}\right.
$$

and for all $x_{0} \in \mathbb{R}$ satisfying

$$
\phi_{*}\left(x_{0}+a\right)-\phi^{*}\left(x_{0}-a\right) \leq \delta,
$$

we have

$$
\operatorname{dist}(\alpha,\{0, b\}+\mathbb{Z})<\varepsilon \quad \text { for all } \alpha \in\left[\phi_{*}\left(x_{0}\right), \phi^{*}\left(x_{0}\right)\right] .
$$

Note that, $\{0, b\}+\mathbb{Z} \equiv \mathbb{Z} \cup(b+\mathbb{Z})$. Roughly speaking, this proposition says that if $\phi$ is flat enough around $x_{0}$, then $\phi\left(x_{0}\right)$ is close to a zero of $f$.

\section{Proof of Proposition 3.2.}

The proof is done by contradiction.

Step 1: construction of a sequence, by contradiction

We assume by contradiction that there exists $\varepsilon_{0}>0$ such that for all $\delta_{n} \rightarrow 0$, there exists $\phi^{n}$ solution of

$$
\left\{\begin{array}{l}
c^{n}\left(\phi^{n}\right)^{\prime}(x)=F\left(\left(\phi^{n}\left(x+r_{i}\right)\right)_{i=0, \ldots, N}\right) \\
\left(\phi^{n}\right)^{\prime} \geq 0 \\
\phi^{n}(x+1) \leq \phi^{n}(x)+1 \\
\left|c^{n}\right| \leq M_{0} \\
\left|c^{n}\left(\phi^{n}\right)^{\prime}\right| \leq M_{0},
\end{array}\right.
$$

such that there exists $x_{n} \in \mathbb{R}$ satisfying

$$
\phi_{*}^{n}\left(x_{n}+a\right)-\left(\phi^{n}\right)^{*}\left(x_{n}-a\right) \leq \delta_{n} \rightarrow 0,
$$

and there exists $\alpha_{n} \in\left[\phi_{*}^{n}\left(x_{n}\right),\left(\phi^{n}\right)^{*}\left(x_{n}\right)\right]$ such that

$$
\operatorname{dist}\left(\alpha_{n},\{0, b\}+\mathbb{Z}\right) \geq \varepsilon_{0}>0 .
$$

Up to replace $\phi^{n}(x)$ by $\phi^{n}\left(x+e_{n}\right)+k_{n}$ with $e_{n} \in \mathbb{R}, k_{n} \in \mathbb{Z}$, we can assume that

$$
\mid \begin{aligned}
& x_{n} \equiv 0 \\
& \phi^{n}(0) \in[0,1) \quad \text { for all } n .
\end{aligned}
$$

Step 2: passing to limit $n \rightarrow+\infty$

Because $\left|c^{n}\right| \leq M_{0}$ then, up to extract a subsequence as $n \rightarrow+\infty$, we have

$$
c^{n} \rightarrow c .
$$


Case 1: $c \neq 0$

For $n$ large enough, we have $\left|c^{n}\right| \geq \frac{|c|}{2} \neq 0$. Hence

$$
\left|\left(\phi^{n}\right)^{\prime}\right| \leq \frac{2 M_{0}}{|c|} \text { for large } n,
$$

thus $\phi^{n}$ is uniformly Lipschitz continuous. Using Ascoli's Theorem and the diagonal extraction argument, $\phi^{n} \rightarrow \phi$ (up to a subsequence) locally uniformly on $\mathbb{R}$. Moreover, $\phi$ satisfies (in the viscosity sense)

$$
\left\{\begin{array}{l}
c \phi^{\prime}(x)=F\left(\left(\phi\left(x+r_{i}\right)\right)_{i=0, \ldots, N}\right) \\
\phi^{\prime} \geq 0
\end{array}\right.
$$

Case 2: $c=0$

Notice that $\phi^{n}(x+1) \leq \phi^{n}(x)+1$ implies (using (3.32))

$$
\begin{cases}\phi^{n}(x) \leq\lceil x\rceil+1 & \text { for } \quad x \geq 0 \\ \phi^{n}(x) \geq-\lceil|x|\rceil & \text { for } \quad x \leq 0 .\end{cases}
$$

Therefore, using Helly's Lemma (Lemma 2.9) and the diagonal extraction argument, $\phi^{n}$ converges (up to a subsequence) to $\phi$ locally a.e. Moreover, we have (using Lemma 2.11 if $\left.c^{n}=0\right)$

$$
c^{n} \int_{b_{1}}^{b_{2}}\left(\phi^{n}\right)^{\prime}(z) d z=\int_{b_{1}}^{b_{2}} F\left(\left(\phi^{n}\left(z+r_{i}\right)\right)_{i=0, \ldots, N}\right) d z
$$

for every $b_{1}<b_{2}$. That is,

$$
c^{n}\left(\phi^{n}\left(b_{2}\right)-\phi^{n}\left(b_{1}\right)\right)=\int_{b_{1}}^{b_{2}} F\left(\left(\phi^{n}\left(z+r_{i}\right)\right)_{i=0, \ldots, N}\right) d z .
$$

But

$$
F\left(\left(\phi^{n}\left(z+r_{i}\right)\right)_{i=0, \ldots, N}\right) \rightarrow F\left(\left(\phi\left(z+r_{i}\right)\right)_{i=0, \ldots, N}\right) \quad \text { a.e. }
$$

and

$$
\left|F\left(\left(\phi^{n}\left(z+r_{i}\right)\right)_{i=0, \ldots, N}\right)\right| \leq m_{0}(1+|z|)
$$

for some constant $m_{0}>0$ (because of (3.34) and the fact that $F$ is globally Lipschitz with $f$ bounded). Thus, using Lebesgue's dominated convergence theorem, we pass to the limit $n \rightarrow+\infty$, and we get

$$
0=\int_{b_{1}}^{b_{2}} F\left(\left(\phi\left(z+r_{i}\right)\right)_{i=0, \ldots, N}\right) d z
$$

which implies (since $b_{1}$ and $b_{2}$ are arbitrary) that

$$
0=F\left(\left(\phi\left(z+r_{i}\right)\right)_{i=0, \ldots, N}\right) \quad \text { a.e. }
$$

Since $\left(\phi^{n}\right)^{\prime} \geq 0$ implies $\phi^{\prime} \geq 0$, then by Lemma $2.11, \phi$ verifies

$$
\left\{\begin{array}{l}
0=F\left(\left(\phi\left(x+r_{i}\right)\right)_{i=0, \ldots, N}\right) \\
\phi^{\prime} \geq 0
\end{array}\right.
$$


in the viscosity sense.

\section{Step 3: getting a contradiction}

Passing to the limit in (3.30) with $x_{n}=0$ implies that

$$
\phi_{*}(a) \leq \phi^{*}(-a) .
$$

But $\phi$ is non-decreasing, then $\phi=$ const $=: k$ over $(-a, a)$. Since $a>r^{*}$, then from (3.33) and (3.35), we get for $x=0$

$$
\begin{aligned}
0 & =F\left(\left(\phi\left(x+r_{i}\right)\right)_{i=0, \ldots, N}\right) \\
& =F\left((k)_{i=0, \ldots, N}\right)=f(k),
\end{aligned}
$$

hence $k \in\{0, b\}+\mathbb{Z}$. On the other hand, since $\alpha_{n} \in\left[\phi_{*}^{n}(0),\left(\phi^{n}\right)^{*}(0)\right]$, then (up to a subsequence)

$$
\alpha_{n} \rightarrow \alpha \in\{k\}=\left[\phi_{*}(0), \phi^{*}(0)\right] .
$$

Moreover, if we pass to limit in (3.31), we get

$$
\operatorname{dist}(\alpha=k,\{0, b\}+\mathbb{Z}) \geq \varepsilon_{0}>0,
$$

which is a contradiction.

\subsection{Proof of Proposition 2.3}

\section{Proof of Proposition 2.3}

The proof is done in several steps.

\section{Step 0: introduction}

Let $p>0$ and $\phi_{p}$ (given by (2.23)) be a non-decreasing solution of

$$
c_{p} \phi_{p}^{\prime}(x)=F\left(\left(\phi_{p}\left(x+r_{i}\right)\right)_{i=0, \ldots, N}\right)
$$

with

$$
\phi_{p}\left(x+\frac{1}{p}\right)=1+\phi_{p}(x)
$$

and $c_{p}$ is given by (2.24). Up to translate $\phi_{p}$, let us suppose that

$$
\left\{\begin{array}{l}
\left(\phi_{p}\right)_{*}(0) \leq b \\
\left(\phi_{p}\right)^{*}(0) \geq b .
\end{array}\right.
$$

Our aim is to pass to limit as $p$ goes to zero.

Step 0.1: introduce $z_{p}$ and $y_{p}$

For any $\varepsilon>0$ small enough $\left(\varepsilon<\frac{1}{2} \min (b, 1-b)\right)$, let $z_{p}, y_{p} \in \mathbb{R}$ such that

$$
\left\{\begin{array}{l}
\left(\phi_{p}\right)^{*}\left(z_{p}\right) \geq b+\varepsilon \\
\left(\phi_{p}\right)_{*}\left(z_{p}\right) \leq b+\varepsilon,
\end{array}\right.
$$

and

$$
\left\{\begin{array}{l}
\left(\phi_{p}\right)^{*}\left(y_{p}\right) \geq b-\varepsilon \\
\left(\phi_{p}\right)_{*}\left(y_{p}\right) \leq b-\varepsilon .
\end{array}\right.
$$


From Proposition 3.2, since $\left(\phi_{p}\right)^{*}\left(z_{p}\right)>b$ and $\left(\phi_{p}\right)_{*}\left(y_{p}\right)<b$, we deduce that (for $a>r^{*}$ )

$$
\left(\phi_{p}\right)_{*}\left(z_{p}+a\right)-\left(\phi_{p}\right)^{*}\left(z_{p}-a\right) \geq \delta(\varepsilon)>0
$$

and

$$
\left(\phi_{p}\right)_{*}\left(y_{p}+a\right)-\left(\phi_{p}\right)^{*}\left(y_{p}-a\right) \geq \delta(\varepsilon)>0
$$

with $\delta(\varepsilon)$ independent of $p$. Moreover, we notice that

$$
y_{p} \leq 0
$$

(Otherwise, $b-\varepsilon \geq\left(\phi_{p}\right)_{*}\left(y_{p}\right) \geq\left(\phi_{p}\right)^{*}(0) \geq b$, a contradiction).

\section{Step 1: viscosity super-solution}

Let

$$
\psi_{p}(x):=\left(\phi_{p}\right)_{*}(x+a)-\left(\phi_{p}\right)^{*}(x-a) .
$$

Notice that $\psi_{p}$ is lower semi continuous and $\psi_{p}(x) \geq 0$ for all $x \in \mathbb{R}$ (because $\left(\phi_{p}\right)_{*}$ is l.s.c, $\left(\phi_{p}\right)^{*}$ is u.s.c and $\phi_{p}$ is non-decreasing). Since (in the viscosity sense)

$$
\left\{\begin{array}{l}
c_{p}\left(\left(\phi_{p}\right)_{*}\right)^{\prime}(x+a) \geq F\left(\left(\left(\phi_{p}\right)_{*}\left(x+a+r_{i}\right)\right)_{i=0, \ldots, N}\right) \\
c_{p}\left(\left(\phi_{p}\right)^{*}\right)^{\prime}(x-a) \leq F\left(\left(\left(\phi_{p}\right)^{*}\left(x-a+r_{i}\right)\right)_{i=0, \ldots, N}\right),
\end{array}\right.
$$

then we can show (using a doubling of variables) the following inequality

$$
c_{p}\left(\psi_{p}\right)_{*}^{\prime}(x) \geq F\left(\left(\left(\phi_{p}\right)_{*}\left(x+a+r_{i}\right)\right)_{i=0, \ldots, N}\right)-F\left(\left(\left(\phi_{p}\right)^{*}\left(x-a+r_{i}\right)\right)_{i=0, \ldots, N}\right),
$$

which holds in the viscosity sense.

Step 2: passing to the limit $p \rightarrow 0$

Since $c_{p}$ is bounded (see Lemma 3.1), then

$$
c_{p} \rightarrow c,
$$

up to a subsequence.

Case 1: $c \neq 0$

For $p$ small enough, we have $\left|c_{p}\right| \geq \frac{|c|}{2} \neq 0$. From (2.26), we deduce that

$$
\left|\phi_{p}^{\prime}\right| \leq \frac{2 M}{|c|} \text { for } p \text { small, }
$$

thus $\phi_{p}$ is uniformly Lipschitz continuous. Using Ascoli's Theorem and the diagonal extraction argument, $\phi_{p} \rightarrow \phi$ (up to a subsequence) locally uniformly on $\mathbb{R}$. Moreover, $\phi$ satisfies, at least in the viscosity sense (using the stability result, Proposition 2.5),

$$
\left\{\begin{array}{l}
c \phi^{\prime}(x)=F\left(\left(\phi\left(x+r_{i}\right)\right)_{i=0, \ldots, N}\right) \\
\phi^{\prime} \geq 0
\end{array}\right.
$$

and

$$
\left\{\begin{array}{l}
(\phi)_{*}(0) \leq b \\
(\phi)^{*}(0) \geq b
\end{array}\right.
$$


Case 2: $c=0$

Let $R>0$ and choose $p$ small enough such that $R<\frac{1}{2 p}$. Since

$$
\phi_{p}\left(\frac{1}{2 p}\right)=1+\phi_{p}\left(\frac{-1}{2 p}\right)
$$

then for all $x \in[-R, R]$, we have

$$
\left|\phi_{p}(x)-\phi_{p}(0)\right| \leq\left|\phi_{p}\left(\frac{1}{2 p}\right)-\phi_{p}\left(\frac{-1}{2 p}\right)\right|=1 .
$$

Notice that (3.36), the monotonicity of $\phi_{p}$ and (3.44) implies that

$$
b-1 \leq \phi_{p}\left(-\frac{1}{2 p}\right) \leq\left(\phi_{p}\right)_{*}(0) \leq b \leq\left(\phi_{p}\right)^{*}(0) \leq \phi_{p}\left(\frac{1}{2 p}\right) \leq b+1,
$$

thus

$$
b-1 \leq \phi_{p}(0) \leq b+1 .
$$

Hence

$$
\left|\phi_{p}\right|_{L^{\infty}[-R, R]} \leq 3
$$

Using Helly's Lemma (Lemma 2.9) and the diagonal extraction argument, $\phi_{p}$ converges locally a.e. (up to a subsequence) to non-decreasing function $\phi$. Thus, $\phi$ satisfies

$$
\left\{\begin{array}{l}
0=c \phi^{\prime}(x)=F\left(\left(\phi\left(x+r_{i}\right)\right)_{i=0, \ldots, N}\right) \\
\phi^{\prime} \geq 0
\end{array}\right.
$$

almost everywhere. Moreover, from Lemma 2.11, we deduce that $\phi$ is a viscosity solution of (3.45) with

$$
\left\{\begin{array}{l}
\phi_{*}(0) \leq b \\
\phi^{*}(0) \geq b
\end{array}\right.
$$

Step 3: first properties of the limit $\phi$ Step 3.1: the oscillation of $\phi$ is bounded

Consider any $R>0$. Choose $p_{0}$ such that $R \leq \frac{1}{2 p_{0}}$ and let $p \in\left(0, p_{0}\right]$. Then

$$
\phi_{p}(R)-\phi_{p}(-R) \leq \phi_{p}\left(\frac{1}{2 p_{0}}\right)-\phi_{p}\left(\frac{-1}{2 p_{0}}\right)=1 .
$$

But $\phi_{p}$ converges (up to a subsequence and at least almost everywhere) to $\phi$, (see Step 2), thus

$$
\phi(R)-\phi(-R) \leq 1
$$

for almost every $R$. Now let $R$ goes to $+\infty$, we conclude that

$$
\phi(+\infty)-\phi(-\infty) \leq 1 .
$$

Step 3.2: $\phi( \pm \infty) \in \mathbb{Z} \cup(\{b\}+\mathbb{Z})$

Since (3.43) is invariant by translation, then

$$
\phi^{n}(x)=\phi(x-n)
$$


is a viscosity solution of

$$
c\left(\phi^{n}\right)^{\prime}(x)=F\left(\left(\phi^{n}\left(x+r_{i}\right)\right)_{i=0, \ldots, N}\right) .
$$

Moreover, $\phi$ is non-decreasing bounded (see Step 3.1), thus $\left(\phi^{n}\right)_{n}$ is a non-increasing sequence of bounded functions. Therefore, $\phi^{n}$ converges pointwise as $n \rightarrow+\infty$. Moreover, since

$$
\lim _{n \rightarrow+\infty}\left(\phi^{n}(x)-\phi(-\infty)\right)=0
$$

then $\phi^{n}$ converges to $\phi(-\infty)$. Now, using the stability for viscosity solutions (see Proposition $2.5)$, we deduce that $\phi(-\infty)$ is a solution of

$$
c(\phi(-\infty))^{\prime}=F\left((\phi(-\infty))_{i=0, \ldots, N}\right)=f(\phi(-\infty)) .
$$

That is

$$
f(\phi(-\infty))=0
$$

Similarly we get $f(\phi(+\infty))=0$. Therefore the assertion of the step follows from $(B)$.

Step 4: $\phi( \pm \infty) \notin\{b\}+\mathbb{Z}$

Since $\phi(+\infty)-\phi(-\infty) \leq 1$ and

$$
\left\{\begin{array}{l}
\phi_{*}(0) \leq b \\
\phi^{*}(0) \geq b
\end{array}\right.
$$

we get that $\phi(-\infty) \in\{b-1,0, b\}$ and $\phi(+\infty) \in\{b, 1, b+1\}$. We want to exclude the cases $\phi( \pm \infty)=b, b \pm 1$. Notice that if $\phi(+\infty)=b+1$, then $\phi(-\infty)=b$. Similarly, if $\phi(-\infty)=b-1$, then $\phi(+\infty)=b$. Therefore, it is sufficient to exclude the cases $\phi( \pm \infty)=b$. At the end, this will show that $\phi(+\infty)=1$ and $\phi(-\infty)=0$.

Suppose to the contrary that

$$
\phi(+\infty)=b,
$$

(the case $\phi(-\infty)=b$ being similar). Let $x_{0}=2 r^{*}$, where $r^{*}=\max _{i=0, \ldots, N}\left|r_{i}\right|$. Since

$$
b=\phi(+\infty) \geq \phi^{*}(0) \geq b,
$$

then $\phi(x)=b$ for all $x>0$. Hence

$$
\phi\left(x_{0}\right)=\phi\left(x_{0} \pm a\right)=b
$$

for $r^{*}<a<2 r^{*}$. Using the uniform convergence of $\phi_{p}$ to $\phi$ (see Lemma 2.10 if $c=0$ ), we deduce that

$$
\phi_{p}\left(x_{0}\right) \rightarrow b
$$

and

$$
\psi_{p}\left(x_{0}\right)=\left(\phi_{p}\right)_{*}\left(x_{0}+a\right)-\left(\phi_{p}\right)^{*}\left(x_{0}-a\right) \rightarrow 0 \quad \text { as } \quad p \rightarrow 0 .
$$

Step 4.1: Equation satisfied by $\psi_{p}$ at its point of minimum

Since (for $z_{p}$ and $y_{p}$ defined in (3.37) and (3.38)) we have

$$
\left\{\begin{array}{lll}
z_{p} \rightarrow+\infty & \text { as } \quad p \rightarrow 0 & (\phi \text { is non-decreasing and } \phi(+\infty)=b) \\
y_{p} \leq 0 & & (\text { by }(3.41)),
\end{array}\right.
$$


then $x_{0} \in\left[y_{p}, z_{p}\right]$ for $p$ small enough. Next, set

$$
m_{p}=\min _{x \in\left[y_{p}, z_{p}\right]} \psi_{p}(x)=\psi_{p}\left(x_{p}^{*}\right) \geq 0 \quad \text { with } \quad x_{p}^{*} \in\left[y_{p}, z_{p}\right]
$$

thus

$$
m_{p}=\psi_{p}\left(x_{p}^{*}\right) \leq \psi_{p}\left(x_{0}\right) \rightarrow 0 \quad \text { as } \quad p \rightarrow 0
$$

In addition, since

$$
\left\{\begin{array}{l}
\psi_{p}\left(y_{p}\right) \geq \delta(\varepsilon)>0 \\
\psi_{p}\left(z_{p}\right) \geq \delta(\varepsilon)>0
\end{array}\right.
$$

then

$$
x_{p}^{*} \in\left(y_{p}, z_{p}\right)
$$

Therefore from (3.42), we get

$$
0=c_{p}\left(\left(\psi_{p}\right)_{*}\right)^{\prime}\left(x_{p}^{*}\right) \geq F\left(\left(\left(\phi_{p}\right)_{*}\left(x_{p}^{*}+a+r_{i}\right)\right)_{i=0, \ldots, N}\right)-F\left(\left(\left(\phi_{p}\right)^{*}\left(x_{p}^{*}-a+r_{i}\right)\right)_{i=0, \ldots, N}\right)
$$

in the viscosity sense (and pointwisely).

Step 4.2: $\psi_{p}\left(x_{p}^{*}+r_{i}\right) \geq \psi_{p}\left(x_{p}^{*}\right)=m_{p}$ for all $i$

Because of (3.47), we have

$$
b-\varepsilon \leq\left(\phi_{p}\right)^{*}\left(y_{p}\right) \leq\left(\phi_{p}\right)^{*}\left(x_{p}^{*}\right) \leq\left(\phi_{p}\right)_{*}\left(z_{p}\right) \leq b+\varepsilon .
$$

Therefore doing a reasoning similar to the one of Step 2, we show that

$$
\phi_{p}\left(x_{p}^{*}+.\right) \rightarrow \phi_{0} \quad \text { a.e. on } \mathbb{R}
$$

and $\phi_{0}$ is a viscosity solution of (3.43). Since

$$
m_{p}=\psi_{p}\left(x_{p}^{*}\right)=\left(\phi_{p}\right)_{*}\left(x_{p}^{*}+a\right)-\left(\phi_{p}\right)^{*}\left(x_{p}^{*}-a\right) \rightarrow 0 \quad \text { as } \quad p \rightarrow 0,
$$

we deduce that

$$
\phi_{0}=\text { const }:=k \quad \text { on } \quad(-a, a)
$$

From Lemma 2.10 and (3.49), we deduce that $k \in[b-\varepsilon, b+\varepsilon]$. Moreover, we have

$$
0=c \phi_{0}^{\prime}(0)=F\left(\left(\phi_{0}\left(0+r_{i}\right)\right)_{i=0, \ldots, N}\right)=f(k),
$$

hence $k=b$. Again, using Lemma 2.10 we deduce that

$$
\sup _{\left(x_{p}^{*}-a+\delta, x_{p}^{*}+a-\delta\right)}\left|\phi_{p}(x)-b\right| \rightarrow 0 \quad \text { for any } \delta>0 .
$$

Moreover, because of (3.50), we can even conclude that

$$
\left(\phi_{p}\right)_{*}\left(x_{p}^{*}+a\right),\left(\phi_{p}\right)^{*}\left(x_{p}^{*}-a\right) \rightarrow b \quad \text { as } \quad p \rightarrow 0 .
$$


Now, since

$$
\left\{\begin{array}{l}
\left(\phi_{p}\right)_{*}\left(y_{p}\right) \leq b-\varepsilon \\
\left(\phi_{p}\right)^{*}\left(z_{p}\right) \geq b+\varepsilon
\end{array}\right.
$$

then $y_{p}, z_{p} \notin\left(x_{p}^{*}-a+\delta, x_{p}^{*}+a-\delta\right)$ for every fixed $\delta$. Since $y_{p}<x_{p}^{*}<z_{p}$, thus choosing $0<\delta \leq a-r^{*}$ implies that

$$
y_{p} \leq x_{p}^{*}+r_{i} \leq z_{p} \text { for all } i
$$

Therefore,

$$
\psi_{p}\left(x_{p}^{*}+r_{i}\right) \geq \psi_{p}\left(x_{p}^{*}\right)=m_{p}
$$

\section{Step 4.3: getting a contradiction}

In this step, we assume that $m_{p}>0$ (it will be shown in Step 5) and we want to get a contradiction. Set

$$
k_{i}= \begin{cases}\left(\phi_{p}\right)_{*}\left(x_{p}^{*}+a+r_{i}\right) & \text { if } \quad r_{i} \leq 0 \\ \left(\phi_{p}\right)^{*}\left(x_{p}^{*}-a+r_{i}\right) & \text { if } \quad r_{i}>0\end{cases}
$$

Hence from (3.53) and using the monotonicity of $F$ together with inequality (3.48), we get

$$
0 \geq F\left(\left(a_{i}\right)_{i=0, \ldots, N}\right)-F\left(\left(c_{i}\right)_{i=0, \ldots, N}\right),
$$

where

$$
a_{i}=\left\{\begin{array}{ll}
k_{i} & \text { if } \quad r_{i} \leq 0 \\
k_{i}+m_{p} & \text { if } \quad r_{i}>0
\end{array} \text { and } c_{i}= \begin{cases}k_{i}-m_{p} & \text { if } r_{i} \leq 0 \\
k_{i} & \text { if } \quad r_{i}>0\end{cases}\right.
$$

Notice that

$$
k_{i} \in\left[\left(\phi_{p}\right)^{*}\left(x_{p}^{*}-a\right),\left(\phi_{p}\right)_{*}\left(x_{p}^{*}+a\right)\right] .
$$

Therefore from (3.52) and the fact that $m_{p} \rightarrow 0$, we deduce that

$$
a_{i} \rightarrow b \quad \text { and } \quad c_{i} \rightarrow b \quad \text { as } \quad p \rightarrow 0 .
$$

Since $F$ is $C^{1}$ near $\{b\}^{N+1}$ and $c_{i}+t\left(a_{i}-c_{i}\right)=c_{i}+t m_{p}$, then

$$
\begin{aligned}
0 & \geq \int_{0}^{1} d t \sum_{i=0}^{N}\left(\left(a_{i}-c_{i}\right) \frac{\partial F}{\partial X_{i}}\left(\left(c_{j}+t\left(a_{j}-c_{j}\right)\right)_{j=0, \ldots, N}\right)\right) \\
& =\int_{0}^{1} d t \sum_{i=0}^{N}\left(m_{p} \frac{\partial F}{\partial X_{i}}\left(\left(c_{j}+t m_{p}\right)_{j=0, \ldots, N}\right)\right) .
\end{aligned}
$$

Since $m_{p}>0$, we get

$$
\begin{aligned}
0 & \geq \int_{0}^{1} d t \sum_{i=0}^{N} \frac{\partial F}{\partial X_{i}}\left(\left(c_{j}+t m_{p}\right)_{j=0, \ldots, N}\right) \\
& =f^{\prime}(b)+\int_{0}^{1} d t\left(\sum_{i=0}^{N} \frac{\partial F}{\partial X_{i}}\left(\left(c_{j}+t m_{p}\right)_{j=0, \ldots, N}\right)-\sum_{i=0}^{N} \frac{\partial F}{\partial X_{i}}(b, \ldots, b)\right) .
\end{aligned}
$$


But $F$ is $C^{1}$ near $\{b\}^{N+1}$ and $c_{i}+t m_{p} \rightarrow b$ for all $i$, thus

$$
\int_{0}^{1} d t\left(\sum_{i=0}^{N} \frac{\partial F}{\partial X_{i}}\left(\left(c_{j}+t m_{p}\right)_{j=0, \ldots, N}\right)-\sum_{i=0}^{N} \frac{\partial F}{\partial X_{i}}(b, \ldots, b)\right) \rightarrow 0 \quad \text { as } \quad p \rightarrow 0 .
$$

This implies that

$$
0 \geq f^{\prime}(b)>0
$$

which is a contradiction because of assumption $(B)$.

Step 5: $m_{p}>0$

We split this step into two cases:

Case 1: $F$ is strongly increasing in some direction

Assume that $F$ verifies in addition:

$$
\frac{\partial F}{\partial X_{i_{1}}} \geq \delta_{0}>0
$$

for certain $i_{1}$ with $r_{i_{1}}>0$ (assuming $r_{i_{1}}<0$ being similar).

Assume to the contrary that $m_{p}=0$. Thus

$$
\psi_{p}\left(x_{p}^{*}\right)=\left(\phi_{p}\right)_{*}\left(x_{p}^{*}+a\right)-\left(\phi_{p}\right)^{*}\left(x_{p}^{*}-a\right)=0 .
$$

Since $\phi_{p}$ is non-decreasing, then

$$
\phi_{p}\left(x_{p}^{*}\right)=\phi_{p_{\left(x_{p}^{*}-a, x_{p}^{*}+a\right)}}=k=\mathrm{const},
$$

where $k$ is a zero of $f$, i.e

$$
f(k)=0 .
$$

Let $d \geq x_{p}^{*}+a$ be the first real number such that

$$
\phi_{p}(d+\eta)>k \text { for every } \eta>0 .
$$

Choose $0<\eta<r_{i_{1}}$ and set

$$
x_{1}=d+\eta-r_{i_{1}} .
$$

From the definition of $d$, we deduce that

$$
\phi_{p}=k \quad \text { on a neighborhood of } x_{1},
$$

hence $\phi_{p}^{\prime}\left(x_{1}\right)=0$. Moreover, we have

$$
\left\{\begin{array}{l}
\phi_{p}\left(x_{1}+r_{i}\right) \geq k \text { for all } i \neq i_{1} \\
\phi_{p}\left(x_{1}+r_{i_{1}}\right)=\phi_{p}(d+\eta)>k \text { for } i=i_{1}
\end{array}\right.
$$

therefore

$$
\begin{aligned}
0=c \phi_{p}^{\prime}\left(x_{1}\right) & =F\left(\left(\phi_{p}\left(x_{1}+r_{i}\right)\right)_{i=0, \ldots, N}\right) \\
& \geq F(k, \ldots, \overbrace{\left.\phi_{p}\left(x_{1}+r_{i_{1}}\right)\right)}^{i_{1}}, \ldots, k) \\
& \geq f(k)+\delta_{0}\left(\phi_{p}(d+\eta)-k\right) \\
& =\delta_{0}\left(\phi_{p}(d+\eta)-k\right)>0,
\end{aligned}
$$


where we have used (3.55) for the last line. This is a contradiction.

\section{Case 2: create the monotonicity}

In fact, we can always assume hypothesis (3.54) for a modification $F_{p}$ of $F$, where

$$
F_{p}\left(X_{0}, X_{1}, \ldots, X_{N}\right)=F\left(X_{0}, X_{1}, \ldots, X_{N}\right)+p\left(X_{i_{1}}-X_{0}\right) .
$$

Then the whole construction works for $F$ replaced by $F_{p}$ with the additional monotonicity property (3.54) with $\delta_{0}=p$. Once we pass to the limit $p \rightarrow 0$, we still get the same contradiction as in Step 4.3 and we recuperate the construction of traveling wave $\phi$ of (2.20) for the function $F$.

\section{Uniqueness of the velocity $c$}

We prove in this section the uniqueness of the velocity of a traveling wave $\phi$ solution of (1.14) (part (a) of Theorem 1.5). We show in the first subsection a comparison principle on the half-line, and we prove the uniqueness of the velocity in the second subsection.

\subsection{Comparison principle on the half-line}

In this subsection, we prove a comparison principle on the half-line that is essentially used to prove the uniqueness of the velocity (in the second subsection of this section) and the uniqueness of the profile $\phi$ that solves (1.14) (in Section 6).

Theorem 4.1 (Comparison principle on $\left(-\infty, r^{*}\right]$ )

Let $F:[0,1]^{N+1} \rightarrow \mathbb{R}$ satisfying $(A)$ and assume that

$$
\mid \begin{aligned}
& \text { there exists } \beta_{0}>0 \text { such that if } \\
& Y=\left(Y_{0}, \ldots, Y_{N}\right), Y+(a, \ldots, a) \in\left[0, \beta_{0}\right]^{N+1} \\
& \text { then } F(Y+(a, \ldots, a))<F(Y) \text { if } a>0 .
\end{aligned}
$$

Let $u, v:\left(-\infty, r^{*}\right] \rightarrow[0,1]$ be respectively a sub and a super-solution of

$$
c u^{\prime}(x)=F\left(\left(u\left(x+r_{i}\right)\right)_{i=0, \ldots, N}\right) \quad \text { on } \quad(-\infty, 0)
$$

in the sense of Definition 2.4. Assume moreover that

$$
u \leq \beta_{0} \quad \text { on } \quad\left(-\infty, r^{*}\right]
$$

and

$$
u \leq v \quad \text { on } \quad\left[0, r^{*}\right] .
$$

Then

$$
u \leq v \text { on }\left(-\infty, r^{*}\right] .
$$

Before giving the proof of this result, we give a corollary which is a comparison principle on $\left[-r^{*},+\infty\right)$. 
Corollary 4.2 (Comparison principle on $\left[-r^{*},+\infty\right)$ )

Let $F:[0,1]^{N+1} \rightarrow \mathbb{R}$ satisfying $(A)$ and assume that:

$$
\mid \begin{aligned}
& \text { there exists } \beta_{0}>0 \text { such that if } \\
& X=\left(X_{0}, \ldots, X_{N}\right), X+(a, \ldots, a) \in\left[1-\beta_{0}, 1\right]^{N+1} \\
& \text { then } F(X+(a, \ldots, a))<F(X) \text { if } a>0 .
\end{aligned}
$$

Let $u, v:\left[-r^{*},+\infty\right) \rightarrow[0,1]$ be respectively a sub and a super-solution of $(4.57)$ on $(0,+\infty)$ in sense of Definition 2.4. Moreover, assume that

$$
v \geq 1-\eta_{0} \text { on }\left[-r^{*},+\infty\right)
$$

and that

$$
u \leq v \quad \text { on } \quad\left[-r^{*}, 0\right]
$$

Then

$$
u \leq v \text { on }\left[-r^{*},+\infty\right)
$$

Remark 4.3 (Inverse monotonicity)

Notice that assumptions (4.56) and (4.58) are satisfied if $F$ is $C^{1}$ on a neighborhood of $\{0\}^{N+1}$ and $\{1\}^{N+1}$ in $[0,1]^{N+1}$ and $f^{\prime}(0)<0, f^{\prime}(1)<0$. This condition means that 0 and 1 are stable equilibria.

Lemma 4.4 (Transformation of a solution of (4.57))

Let $u, v:\left(-\infty, r^{*}\right] \rightarrow[0,1]$ be respectively a sub and super-solution of (4.57) in the sense of Definition 2.4. Then

$$
\widehat{u}(x):=1-u(-x) \text { and } \widehat{v}(x):=1-v(-x)
$$

are respectively a super and a sub-solution of $(4.57)$ on $\left[-r^{*},+\infty\right)$ with $F, c$ and $r_{i}$ (for all $i \in\{0, \ldots, N\})$ replaced by $\widehat{F}, \widehat{c}$ and $\widehat{r}_{i}$, given by

$$
\left\{\begin{array}{l}
\widehat{F}\left(X_{0}, \ldots, X_{N}\right)=-F\left(1-X_{0}, \ldots, 1-X_{N}\right) \\
\widehat{c}:=-c \\
\widehat{r}_{i}:=-r_{i} .
\end{array}\right.
$$

Moreover,

$$
\widehat{F}:[0,1]^{N+1} \rightarrow \mathbb{R}
$$

satisfies $(A),(B)$ and $(C)$, where $b$ and $f$ are replaced by

$$
\left\{\begin{array}{l}
\widehat{b}:=1-b \\
\widehat{f}(v):=-f(-v)
\end{array}\right.
$$

in $(B)$.

Notice that, Lemma 4.4 is still true even though $u, v: \mathbb{R} \rightarrow[0,1]$ are a sub and a supersolution of (4.57) on $\mathbb{R}$. 


\section{Proof of Lemma 4.4}

Let $u:\left(-\infty, r^{*}\right] \rightarrow[0,1]$ be a sub-solution of $(4.57)$ and set $\widehat{u}(x)=1-u(-x)$. It is then easy to see that in the viscosity sense

$$
\begin{aligned}
c \widehat{u}^{\prime}(x)=c u^{\prime}(-x) & \leq F\left(\left(u\left(-x+r_{i}\right)\right)_{i=0, \ldots, N}\right) \\
& =F\left(\left(1-\widehat{u}\left(x-r_{i}\right)\right)_{i=0, \ldots, N}\right) .
\end{aligned}
$$

Hence $\widehat{u}$ is a super-solution of (4.57) on $\left[-r^{*},+\infty\right)$ with $F, r_{i}$ and $c$ replaced by $\widehat{F}, \widehat{r}_{i}:=-r_{i}$ and $\widehat{c}:=-c$ given in (4.59). Similarly, we show that $\widehat{v}$ is a sub-solution of the same equation on $\left[-r^{*},+\infty\right)$.

\section{Proof of Corollary 4.2.}

Let $u, v:\left[-r^{*},+\infty\right) \rightarrow[0,1]$ be a sub and super-solution of $(4.57)$ on $(0,+\infty)$ such that $v \geq 1-\beta_{0}$ on $\left[-r^{*},+\infty\right)$. We set $\widehat{u}(x)=1-u(-x)$ and $\widehat{v}(x)=1-v(-x)$. It is then easy to see that $\widehat{u}, \widehat{v} \in[0,1], \widehat{v} \leq \beta_{0}$ on $\left(-\infty, r^{*}\right]$.

Using Lemma 4.4, we show that $\widehat{u}$ and $\widehat{v}$ are respectively a super and a sub-solution of (4.57) with $\left(F, c, r_{i}\right)$ replaced by $\left(\widehat{F}, \widehat{c}, \widehat{r}_{i}\right)$ defined in (4.59). Moreover, using the fact that $F$ satisfies (4.58), we deduce that $\widehat{F}$ satisfies (4.56).

We then deduce by Theorem 4.1 that

$$
\widehat{v} \leq \widehat{u} \quad \text { on } \quad\left(-\infty, r^{*}\right]
$$

i.e.

$$
u \leq v \quad \text { on } \quad\left[-r^{*},-\infty\right)
$$

We now turn to the proof of Theorem 4.1.

\section{Proof of Theorem 4.1.}

Let $u, v:\left(-\infty, r^{*}\right] \rightarrow[0,1]$ be respectively a sub and a super-solution of (4.57) such that

$$
u \leq \beta_{0} \quad \text { on } \quad\left(-\infty, r^{*}\right]
$$

and $u \leq v$ on $\left[0, r^{*}\right]$.

\section{Step 0: Introduction}

Set

$$
\bar{v}:=\min \left(v, \beta_{0}\right)
$$

According to (4.56) we have

$$
F\left(\beta_{0}, \ldots, \beta_{0}\right)<F(0, \ldots, 0)=f(0)=0
$$

thus the constant $\beta_{0}$ is a super-solution of (4.57). Hence $\bar{v}$ is a super-solution of (4.57) on $(-\infty, 0)$ with $u \leq \bar{v}$ on $\left[0, r^{*}\right]$. Moreover, since $\bar{v} \leq v$, it is sufficient to prove the comparison principle (Theorem 4.1) between $u$ and $\bar{v}$ which satisfy in addition $u, \bar{v} \in\left[0, \beta_{0}\right]$.

For simplicity, we note $\bar{v}$ as $v$ with $u, v \in\left[0, \beta_{0}\right]$ and $u \leq v$ on $\left[0, r^{*}\right]$.

\section{Step 1: Doubling the variables}

Suppose by contradiction that

$$
M=\sup _{x \in\left(-\infty, r^{*}\right]} u(x)-v(x)>0 .
$$


Let $\varepsilon, \alpha>0$ and define

$$
\begin{aligned}
M_{\varepsilon, \alpha} & :=\sup _{x, y \in\left(-\infty, r^{*}\right]}\left(u(x)-v(y)-\frac{|x-y|^{2}}{2 \varepsilon}-\alpha|x|^{2}\right) \\
& =u\left(\bar{x}_{\varepsilon}\right)-v\left(\bar{y}_{\varepsilon}\right)-\frac{\left|\bar{x}_{\varepsilon}-\bar{y}_{\varepsilon}\right|^{2}}{2 \varepsilon}-\alpha\left|\bar{x}_{\varepsilon}\right|^{2},
\end{aligned}
$$

for certain $\bar{x}_{\varepsilon}, \bar{y}_{\varepsilon} \in\left(-\infty,-r^{*}\right]$. Note that the maximum is reached since the function

$$
(x, y) \mapsto \psi(x, y)=u(x)-v(y)-\frac{|x-y|^{2}}{2 \varepsilon}-\alpha|x|^{2}
$$

is upper semi-continuous and satisfies $\psi(x, y) \rightarrow-\infty$ as $|x|,|y| \rightarrow+\infty$. Moreover, for all $\delta>0$, there exists $x_{\delta} \in\left(-\infty, r^{*}\right]$ such that

$$
M \geq u\left(x_{\delta}\right)-v\left(x_{\delta}\right) \geq M-\delta .
$$

Hence

$$
\begin{aligned}
M_{\varepsilon, \alpha} & \geq u\left(x_{\delta}\right)-v\left(x_{\delta}\right)-\alpha\left|x_{\delta}\right|^{2} \\
& \geq M-\delta-\alpha\left|x_{\delta}\right|^{2} \\
& \geq \frac{M}{2}>0
\end{aligned}
$$

for $\delta=\frac{M}{4}$ and $\alpha$ chosen small enough such that $\alpha<\frac{M}{4\left|x_{\delta}\right|^{2}}$. Moreover, since $u\left(\bar{x}_{\varepsilon}\right)-v\left(\bar{y}_{\varepsilon}\right) \leq$ $\beta_{0}$, we have

$$
\frac{\left|\bar{x}_{\varepsilon}-\bar{y}_{\varepsilon}\right|^{2}}{2 \varepsilon}+\alpha\left|\bar{x}_{\varepsilon}\right|^{2} \leq \beta_{0} .
$$

Step 2: There exists $\alpha$ small enough and $\varepsilon \rightarrow 0$ such that $\bar{x}_{\varepsilon} \in\left[0, r^{*}\right]$ or $\bar{y}_{\varepsilon} \in\left[0, r^{*}\right]$ Assume that $\bar{x}_{\varepsilon} \in\left[0, r^{*}\right]$ (the case $\bar{y}_{\varepsilon} \in\left[0, r^{*}\right]$ being similar). Using (4.60), we deduce that $\bar{y}_{\varepsilon} \in\left[-\sqrt{2 \beta_{0} \varepsilon}, r^{*}\right]$. Then $\bar{x}_{\varepsilon}$ and $\bar{y}_{\varepsilon}$ converge (up to a subsequence) to a certain $\bar{x}_{0} \in\left[0, r^{*}\right]$ as $\varepsilon \rightarrow 0$ (from (4.60), the two limits coincide). We then deduce that

$$
\begin{aligned}
0<\frac{M}{2} & \leq \limsup _{\varepsilon \rightarrow 0}\left(u\left(\bar{x}_{\varepsilon}\right)-v\left(\bar{y}_{\varepsilon}\right)\right) \\
& \leq u\left(\bar{x}_{0}\right)-v\left(\bar{x}_{0}\right) \leq 0,
\end{aligned}
$$

which is a contradiction. The last inequality takes place since $u \leq v$ on $\left[0, r^{*}\right]$.

Step 3: For all $\alpha$ and $\varepsilon$ small enough, we have $\bar{x}_{\varepsilon}, \bar{y}_{\varepsilon} \in(-\infty, 0)$ Step 3.1: Viscosity inequalities

We have

$$
u(x) \leq v\left(\bar{y}_{\varepsilon}\right)+M_{\varepsilon, \alpha}+\frac{\left|x-\bar{y}_{\varepsilon}\right|^{2}}{2 \varepsilon}+\alpha|x|^{2}:=\phi(x),
$$

and $u\left(\bar{x}_{\varepsilon}\right)=\phi\left(\bar{x}_{\varepsilon}\right)$. Thus

$$
c\left(\frac{\bar{x}_{\varepsilon}-\bar{y}_{\varepsilon}}{\varepsilon}+2 \alpha \bar{x}_{\varepsilon}\right)=c \phi^{\prime}\left(\bar{x}_{\varepsilon}\right) \leq F\left(\left(u\left(\bar{x}_{\varepsilon}+r_{i}\right)\right)_{i=0, \ldots, N}\right) .
$$


Similarly, we get

$$
c\left(\frac{\bar{x}_{\varepsilon}-\bar{y}_{\varepsilon}}{\varepsilon}\right) \geq F\left(\left(v\left(\bar{y}_{\varepsilon}+r_{i}\right)\right)_{i=0, \ldots, N}\right) .
$$

Subtracting (4.62) from (4.61) implies that

$$
2 c \alpha \bar{x}_{\varepsilon} \leq F\left(\left(u\left(\bar{x}_{\varepsilon}+r_{i}\right)\right)_{i=0, \ldots, N}\right)-F\left(\left(v\left(\bar{y}_{\varepsilon}+r_{i}\right)\right)_{i=0, \ldots, N}\right) .
$$

Note that from (4.60)

$$
\alpha\left|\bar{x}_{\varepsilon}\right| \leq \sqrt{\alpha \beta_{0}}
$$

This implies that for $\varepsilon$ fixed, $\alpha \bar{x}_{\varepsilon} \rightarrow 0$ as $\alpha \rightarrow 0$.

Step 3.2: Passing to the limit $\alpha \rightarrow 0$

We have

$$
u(x)-v(y)-\frac{|x-y|^{2}}{2 \varepsilon}-\alpha|x|^{2} \leq u\left(\bar{x}_{\varepsilon}\right)-v\left(\bar{y}_{\varepsilon}\right)-\frac{\left|\bar{x}_{\varepsilon}-\bar{y}_{\varepsilon}\right|^{2}}{2 \varepsilon}-\alpha\left|\bar{x}_{\varepsilon}\right|^{2} .
$$

Set

$$
\mid \begin{aligned}
& u_{i}^{\alpha}=u\left(\bar{x}_{\varepsilon}+r_{i}\right) \\
& v_{i}^{\alpha}=v\left(\bar{y}_{\varepsilon}+r_{i}\right)
\end{aligned}
$$

then

$$
\begin{cases}u_{i}^{\alpha} \leq v_{i}^{\alpha}+m_{\alpha}+\delta_{i}^{\alpha} & \text { if } \quad i \neq 0 \\ u_{0}^{\alpha}=v_{0}^{\alpha}+m_{\alpha} & \text { if } \quad i=0\end{cases}
$$

where $m_{\alpha}=u_{0}^{\alpha}-v_{0}^{\alpha}$ and $\delta_{i}^{\alpha}=2 \alpha \bar{x}_{\varepsilon} r_{i}+\alpha\left|r_{i}\right|^{2}$. For $\varepsilon$ fixed, since $u_{i}^{\alpha}, v_{i}^{\alpha} \in\left[0, \beta_{0}\right]$ and $\frac{M}{2} \leq m_{\alpha} \leq \beta_{0}$, we deduce that as $\alpha \rightarrow 0$ and up to a subsequence,

$$
\left\{\begin{array}{l}
u_{i}^{\alpha} \rightarrow u_{i}^{0} \\
v_{i}^{\alpha} \rightarrow v_{i}^{0} \\
m_{\alpha} \rightarrow m_{0} \\
\delta_{i}^{\alpha} \rightarrow 0
\end{array}\right.
$$

with $u_{i}^{0}, v_{i}^{0} \in\left[0, \beta_{0}\right], 0<\frac{M}{2} \leq m_{0} \leq \beta_{0}$ and

$$
\begin{cases}u_{i}^{0} \leq v_{i}^{0}+m_{0} & \text { if } \quad i \neq 0 \\ u_{0}^{0}=v_{0}^{0}+m_{0} & \text { if } \quad i=0 .\end{cases}
$$

Moreover, passing to the limit in (4.63) implies that

$$
0 \leq F\left(\left(u_{i}^{0}\right)_{i=0, \ldots, N}\right)-F\left(\left(v_{i}^{0}\right)_{i=0, \ldots, N}\right)
$$

\section{Step 4: Getting a contradiction}

We claim that for all $i$, there exists $l_{i}, l_{i}^{\prime} \geq 0$ such that

$$
u_{i}^{0}+l_{i}=v_{i}^{0}-l_{i}^{\prime}+m_{0},
$$


and

$$
\left\{\begin{array}{l}
\bar{u}_{i}^{0}:=u_{i}^{0}+l_{i} \leq \beta_{0} \\
\bar{v}_{i}^{0}:=v_{i}^{0}-l_{i}^{\prime} \geq 0 .
\end{array}\right.
$$

Recall that for all $i \in\{0, \ldots, N\}$, we have

$$
\left\{\begin{array}{l}
u_{i}^{0}, v_{i}^{0} \in\left[0, \beta_{0}\right] \\
u_{i}^{0} \leq v_{i}^{0}+m_{0} \\
u_{0}^{0}-v_{0}^{0}=m_{0} \leq \beta_{0} .
\end{array}\right.
$$

If for some $i, u_{i}^{0}=v_{i}^{0}+m_{0}$, then it suffices to take $l_{i}=l_{i}^{\prime}=0$. Assume then that $u_{i}^{0}<v_{i}^{0}+m_{0}$.

Case 1: $u_{i}^{0}, v_{i}^{0} \in\left(v_{0}^{0}, u_{0}^{0}\right)$

Set $l_{i}=u_{0}^{0}-u_{i}^{0}$ and $l_{i}^{\prime}=v_{i}^{0}-v_{0}^{0}$. Then

$$
\left\{\begin{array}{l}
\bar{u}_{i}^{0}=u_{i}^{0}+l_{i}=u_{0}^{0} \leq \beta_{0} \\
\bar{v}_{i}^{0}=v_{i}^{0}-l_{i}^{\prime}=v_{0}^{0} \geq 0
\end{array}\right.
$$

and $\bar{u}_{i}^{0}=\bar{v}_{i}^{0}+m_{0}$.

Case $2: u_{i}^{0}>u_{0}^{0}$ and $v_{i}^{0}>v_{0}^{0}$

Since $u_{i}^{0}-v_{0}^{0}>m_{0}$, then there exists $l_{i}^{\prime}<v_{i}^{0}-v_{0}^{0}$ such that

$$
u_{i}^{0}=v_{i}^{0}-l_{i}^{\prime}+m_{0}
$$

and $\bar{v}_{i}^{0}=v_{i}^{0}-l_{i}^{\prime}>v_{0}^{0} \geq 0$. Thus, it is sufficient to take $l_{i}=0$.

Case 3: $u_{i}^{0}<u_{0}^{0}$ and $v_{i}^{0}<v_{0}^{0}$

This case can be treated as Case 2 by taking $l_{i}^{\prime}=0$ and $l_{i}<u_{0}^{0}-u_{i}^{0}$.

Finally, going back to $(4.64)$, since $F$ is non-decreasing, we deduce that

$$
\begin{aligned}
0 & \leq F\left(\left(u_{i}^{0}\right)_{i=0, \ldots, N}\right)-F\left(\left(v_{i}^{0}\right)_{i=0, \ldots, N}\right) \\
& \leq F\left(\left(\bar{u}_{i}^{0}\right)_{i=0, \ldots, N}\right)-F\left(\left(\bar{v}_{i}^{0}\right)_{i=0, \ldots, N}\right) \\
& =F\left(\left(\bar{u}_{i}^{0}\right)_{i=0, \ldots, N}\right)-F\left(\left(\bar{u}_{i}^{0}-m_{0}\right)_{i=0, \ldots, N}\right) \\
& <0 .
\end{aligned}
$$

Last inequality takes place since $F$ verifies (4.56) for $\bar{u}_{i}^{0}, \bar{u}_{i}^{0}-m_{0} \in\left[0, \beta_{0}\right]$ and $m_{0}>0$. Therefore, we get a contradiction.

\subsection{Uniqueness of the velocity}

This subsection is devoted to prove the uniqueness of the velocity $c$ of a traveling wave that solves (1.14).

\section{Proposition 4.5 (Uniqueness of $c$ )}

Under assumptions $(A)$, consider the function $F$ defined on $[0,1]^{N+1}$. Let $\left(c_{j}, \phi_{j}\right)$ be a solution of (1.14) for $j=1,2$. If $F$ satisfies in addition $(C)$, then $c_{1}=c_{2}$. 


\section{Proof of Proposition 4.5.}

Suppose that for $j=1,2,\left(c_{j}, \phi_{j}\right)$ is a solution of (1.14) and assume by contradiction that $c_{1}<c_{2}$. We have,

$$
\phi_{j}(-\infty)=0 \quad \text { and } \quad \phi_{j}(+\infty)=1 .
$$

We set $\delta=\min \left(\beta_{0}, \frac{1}{4}\right)$ where $\beta_{0}$ is given in assumption $(C)$. Up to translate $\phi_{1}$ and $\phi_{2}$, we can assume that

$$
\phi_{1}(x) \geq 1-\delta \quad \forall x \geq-r^{*}
$$

and

$$
\phi_{2}(x) \leq \delta \quad \forall x \leq r^{*}
$$

This implies that

$$
\phi_{2} \leq \phi_{1} \text { over }\left[-r^{*}, r^{*}\right] .
$$

Moreover, since $c_{1}<c_{2}$, we have

$$
c_{1} \phi_{2}^{\prime}(x) \leq c_{2} \phi_{2}^{\prime}(x)=F\left(\left(\phi_{2}\left(x+r_{i}\right)\right)_{i=0, \ldots, N}\right) .
$$

Hence $\left(c_{1}, \phi_{2}\right)$ is a sub-solution of (1.14). Since

$$
\phi_{1} \geq 1-\delta \quad \text { on } \quad\left[-r^{*},+\infty\right),
$$

we deduce using Corollary 4.2 that

$$
\phi_{2} \leq \phi_{1} \text { over }\left[-r^{*},+\infty\right)
$$

Similarly, since

$$
\phi_{2} \leq \delta \quad \text { on } \quad\left(-\infty, r^{*}\right]
$$

we deduce using Theorem 4.1 that

$$
\phi_{2} \leq \phi_{1} \text { over }\left(-\infty, r^{*}\right]
$$

Therefore,

$$
\phi_{2} \leq \phi_{1} \text { over } \mathbb{R}
$$

Next, set

$$
\mid \begin{aligned}
& u_{1}(t, x)=\phi_{1}\left(x+c_{1} t\right) \\
& u_{2}(t, x)=\phi_{2}\left(x+c_{2} t\right)
\end{aligned}
$$

then for $j=1,2$, we have

$$
\partial_{t} u_{j}(t, x)=F\left(\left(u_{j}\left(t, x+r_{i}\right)\right)_{i=0, \ldots, N}\right) .
$$

Moreover, at time $t=0$, we have

$$
u_{1}(0, x)=\phi_{1}(x) \geq \phi_{2}(x)=u_{2}(0, x) \text { over } \mathbb{R},
$$

thus applying the comparison principle for equation (4.66) (see [23]), we get

$$
u_{1} \geq u_{2} \quad \forall t \geq 0 \forall x \in \mathbb{R} .
$$


Taking $x=y-c_{1} t$, we get

$$
\phi_{1}(y) \geq \phi_{2}\left(y+\left(c_{2}-c_{1}\right) t\right), \quad \forall t \geq 0, \forall y \in \mathbb{R} .
$$

Using that $c_{1}<c_{2}$, and passing to the limit $t \rightarrow+\infty$, we get

$$
\phi_{1}(y) \geq \phi_{2}(+\infty)=1, \quad \forall y \in \mathbb{R} .
$$

But $\phi_{1}(-\infty)=0$, hence a contradiction. Therefore $c_{1} \geq c_{2}$. Similarly, we show that $c_{2} \geq c_{1}$, hence $c_{1}=c_{2}$.

\section{Asymptotics for the profile}

In this section, our main result is the asymptotics near $\pm \infty$ for solutions $\phi: \mathbb{R} \rightarrow[0,1]$ of

$$
c \phi^{\prime}(x)=F\left(\left(\phi\left(x+r_{i}\right)\right)_{i=0, \ldots, N}\right) \quad \text { on } \quad \mathbb{R},
$$

namely Proposition 5.1.

Proposition 5.1 (Asymptotics near $\pm \infty$ )

Consider a function $F$ defined on $[0,1]^{N+1}$ satisfying $(A)$ and $(C)$, and assume that $c \neq 0$. Then

i) asymptotics near $-\infty$

Let $\phi: \mathbb{R} \rightarrow[0,1]$ be a solution of (5.67), satisfying

$$
\phi(-\infty)=0 \quad \text { and } \quad \phi \geq \delta>0 \quad \text { on }\left[0, r^{*}\right]
$$

for some $\delta>0$ and assume $(E+)$ ii). If there exists a unique $\lambda^{+}>0$ solution of

$$
c \lambda=\sum_{i=0}^{N} \frac{\partial F}{\partial X_{i}}(0, \ldots, 0) e^{\lambda r_{i}}
$$

then for any sequence $\left(x_{n}\right)_{n}, x_{n} \rightarrow-\infty$, there exists a subsequence $\left(x_{n^{\prime}}\right)_{n^{\prime}}$ and $A>0$ such that

$$
\frac{\phi\left(x+x_{n^{\prime}}\right)}{e^{\lambda^{+} x_{n^{\prime}}}} \longrightarrow A e^{\lambda^{+} x} \quad \text { locally uniformly on } \mathbb{R} \text { as } n^{\prime} \rightarrow+\infty \text {. }
$$

ii) asymptotics near $+\infty$

Let $\phi: \mathbb{R} \rightarrow[0,1]$ be a solution of (5.67), satisfying

$$
\phi(+\infty)=1 \quad \text { and } \quad \phi \leq 1-\delta<1 \quad \text { on } \quad\left[0, r^{*}\right]
$$

for some $\delta>0$ and assume $(E-)$ ii). If there exists a unique $\lambda^{-}<0$ solution of

$$
c \lambda=\sum_{i=0}^{N} \frac{\partial F}{\partial X_{i}}(1, \ldots, 1) e^{\lambda r_{i}}
$$

then for any sequence $\left(x_{n}\right)_{n}, x_{n} \rightarrow+\infty$, there exists a subsequence $\left(x_{n^{\prime}}\right)_{n^{\prime}}$ and $A>0$ such that

$$
\frac{1-\phi\left(x+x_{n^{\prime}}\right)}{e^{\lambda^{-} x_{n^{\prime}}}} \longrightarrow A e^{\lambda^{-} x} \quad \text { locally uniformly on } \mathbb{R} \text { as } n^{\prime} \rightarrow+\infty \text {. }
$$




\subsection{Uniqueness and existence of $\lambda^{ \pm}$}

In this subsection, we address the question of the existence and uniqueness of $\lambda^{ \pm}$.

\section{Lemma 5.2 (Uniqueness and existence of $\lambda^{+}$)}

Assume $(A)$ and suppose that $\nabla F(0)$ exists with $f^{\prime}(0)<0$. Then there is at most one solution $\lambda^{+}>0$ of (5.68). Moreover, if $c<0$ or if we assume $\left.(E+) i\right)$, then there exists a (unique) solution $\lambda^{+}>0$ of (5.68).

\section{Proof of Lemma 5.2}

Step 1: Uniqueness

Let

$$
g(\lambda):=\sum_{i=0}^{N} \frac{\partial F}{\partial X_{i}}(0, \ldots, 0) e^{\lambda r_{i}}-c \lambda .
$$

Because of assumption $(A)$, the function $g$ is convex and

$$
g(0)=f^{\prime}(0)<0 .
$$

Thus, there exists at most one solution $\lambda^{+}>0$ of (5.68) and if $\lambda^{+}$exists, then we have

$$
g<0 \quad \text { on }\left(0, \lambda^{+}\right) \text {and } g>0 \text { on }\left(\lambda^{+},+\infty\right) .
$$

\section{Step 2: Existence}

Assume $c<0$. We have

$$
g(\lambda) \geq \frac{\partial F}{\partial X_{0}}(0, \ldots, 0)-c \lambda,
$$

which implies that $\lim _{\lambda \rightarrow+\infty} g(\lambda)=+\infty$. On the other hand, if we assume $\left.(E+) i\right)$, then

$$
g(\lambda) \geq \frac{\partial F}{\partial X_{0}}(0, \ldots, 0)+\frac{\partial F}{\partial X_{i_{+}}}(0, \ldots, 0) e^{\lambda r_{i_{+}}}-c \lambda,
$$

which implies that $\lim _{\lambda \rightarrow+\infty} g(\lambda)=+\infty$.

Therefore, there exists a unique $\lambda^{+}>0$ such that $g\left(\lambda^{+}\right)=0$.

In the same way (or using Lemma 4.4), we can prove the following lemma concerning $\lambda^{-}$

Lemma 5.3 (Uniqueness and existence of $\lambda^{-}$)

Assume $(A)$ and suppose that $\nabla F(1, \ldots, 1)$ exists with $f^{\prime}(1)<0$. Then there is at most one solution $\lambda^{-}<0$ of (5.69). Moreover, if $c>0$ or if we assume $\left.(E-) i\right)$, then there exists a (unique) solution $\lambda^{-}<0$ of (5.69). 


\subsection{Proof of Proposition 5.1}

In this subsection, we prove that any solution of (5.67) is exponentially bounded (from above and below) near $-\infty$. Finally, we prove Proposition $5.1 \mathrm{i}$ ).

Lemma 5.4 (Exponential bounds for a solution of $(5.67)$ near $-\infty$ )

Assume $(A),(C)$ and $(E+)$ ii $)$. Let $\phi:(-\infty, 0] \rightarrow[0,1]$ be a solution of $(5.67)$ on $\left(-\infty,-r^{*}\right)$ satisfying $\phi(-\infty)=0$ and assume that there exists $\lambda^{+}>0$ solution of (5.68). Then there exists $k_{2}$ such that

$$
\phi(x) \leq k_{2} e^{\lambda^{+} x} \quad \text { for all } \quad x \leq 0
$$

Moreover, if

$$
\phi \geq \delta>0 \text { on }\left[-r^{*}, 0\right] \text { for some } \delta>0,
$$

then there exists $k_{1}>0$ such that

$$
k_{1} e^{\lambda^{+} x} \leq \phi(x) \quad \text { for all } \quad x \leq 0
$$

Remark 5.5 Notice that the exponential bounds of Lemma 5.4 do not holds if we do not assume $(E+)$ ii). To see this, it suffices to define $f(u)=-u^{\prime}$ with $u(x)=-x e^{x}$. A simple computation then gives that

$$
\left\{\begin{array}{l}
f(0)=0 \\
f^{\prime}(0)=-1 \\
f^{\prime}(u)-f^{\prime}(0) \sim_{u \rightarrow 0} \frac{-1}{\ln u}
\end{array}\right.
$$

and so $f$ does not satisfies $(E+)$ ii) and $u$ is not exponentially bounded.

\section{Proof of Lemma 5.4}

The idea of the proof is to construct a sub and super-solution of

$$
c \phi^{\prime}(x)=F\left(\left(\phi\left(x+r_{i}\right)\right)_{i=0, \ldots, N}\right) \quad \text { on } \quad\left(-\infty,-r^{*}\right)
$$

then, using the comparison principle (Theorem 4.1), we deduce the existence of $k_{1}$ and $k_{2}$. Let $\lambda^{+}>0$ be the solution of (5.68) and consider the perturbation $\lambda^{+}<\lambda^{\prime}<(1+\alpha) \lambda^{+}$ with $\alpha$ given in assumption $(E+) i i)$.

Step 1: existence of $k_{1}$ Step 1.1: construction of a sub-solution of (5.73)

Set

$$
\underline{\phi}(x)=A\left(e^{\lambda^{+} x}+e^{\lambda^{\prime} x}\right)
$$

defined on $(-\infty, 0]$, where $A>0$ will be chosen such that $\phi$ is a sub-solution of (5.73). Since $\lambda^{+}$is a solution of (5.68), then for $x \in\left(-\infty,-r^{*}\right)$ we have

$$
\begin{aligned}
c \underline{\phi^{\prime}}(x) & =c \lambda^{+} A e^{\lambda^{+} x}+c A \lambda^{\prime} e^{\lambda^{\prime} x} \\
& =\nabla F(0, \ldots, 0) \cdot\left(\left(A e^{\lambda^{+}\left(x+r_{i}\right)}\right)_{i=0, \ldots, N}\right)+c A \lambda^{\prime} e^{\lambda^{\prime} x} \\
& =\nabla F(0, \ldots, 0) \cdot\left(\left(\underline{\phi}\left(x+r_{i}\right)\right)_{i=0, \ldots, N}\right)-A e^{\lambda^{\prime} x}\left(\nabla F(0, \ldots, 0) \cdot\left(\left(e^{\lambda^{\prime} r_{i}}\right)_{i=0, \ldots, N}\right)-c \lambda^{\prime}\right) \\
& \leq F\left(\left(\underline{\phi}\left(x+r_{i}\right)\right)_{i=0, \ldots, N}\right)+C_{0}|\Phi(x)|^{1+\alpha}-A e^{\lambda^{\prime} x} g\left(\lambda^{\prime}\right),
\end{aligned}
$$


where for the last line we have used $(E+) i i), \Phi(x)=\left(\left(\phi\left(x+r_{i}\right)\right)_{i=0, \ldots, N}\right)$ and $g$ defined in (5.70). Using the fact that for $x \in\left(-\infty,-r^{*}\right)$, we have $\underline{\phi}\left(x+r_{i}\right) \leq 2 A e^{\lambda^{+}\left(x+r^{*}\right)}$. We get

$$
\begin{aligned}
c \underline{\phi}^{\prime}(x)-F\left(\left(\underline{\phi}\left(x+r_{i}\right)\right)_{i=0, \ldots, N}\right) & \leq A\left(2^{1+\alpha} C_{0} A^{\alpha} e^{(1+\alpha) \lambda^{+}\left(x+r^{*}\right)}|E|^{1+\alpha}-e^{\lambda^{\prime} x} g\left(\lambda^{\prime}\right)\right) \\
& \leq A\left(2^{1+\alpha} C_{0} A^{\alpha} e^{(1+\alpha) \lambda^{+} r^{*}}|E|^{1+\alpha}-g\left(\lambda^{\prime}\right)\right) e^{\lambda^{\prime} x}
\end{aligned}
$$

with $E=(1, \ldots, 1) \in \mathbb{R}^{N+1}$. Since $g\left(\lambda^{\prime}\right)>0($ see $(5.71))$,

$$
c \underline{\phi^{\prime}}(x) \leq F\left(\left(\underline{\phi}\left(x+r_{i}\right)\right)_{i=0, \ldots, N}\right) \leq 0 \quad \text { for } A \text { small enough. }
$$

This shows that $\phi$ is a sub-solution of $(5.73)$ on $\left(-\infty,-r^{*}\right)$.

\section{Step 1.2: applying the comparison principle}

Up to decrease $A>0$, let us assume moreover that $2 A \leq \min \left(\delta, \beta_{0}\right)$ with $\delta$ given in $(5.72)$ and $\beta_{0}$ given in assumption $(C)$ (this is possible since $A$ can be chosen as small as we want). Thus

$$
\phi \geq \delta \geq 2 A \geq \underline{\phi} \quad \text { on } \quad\left[-r^{*}, 0\right]
$$

and

$$
\underline{\phi} \leq 2 A \leq \beta_{0} \quad \text { on } \quad(-\infty, 0] .
$$

Hence using the comparison principle (Theorem 4.1 and a shift of the functions), we deduce that

$$
\underline{\phi}(x) \leq \phi(x) \text { for all } x \leq 0 \text {. }
$$

This implies that $\phi$ satisfies

$$
k_{1}:=A \leq \frac{\phi(x)}{e^{\lambda^{+} x}} \quad \text { for all } \quad x \leq 0
$$

\section{Step 2: existence of $k_{2}$}

Step 2.1: construction of a super-solution of (5.73)

Define for $x \in(-\infty, 0]$ the function

$$
\bar{\phi}(x)=A\left(2 e^{\lambda^{+} x}-e^{\lambda^{\prime} x}\right)
$$

Repeating the same proof as in Step 1, we get

$$
\begin{aligned}
c \bar{\phi}^{\prime}(x)-F\left(\left(\bar{\phi}\left(x+r_{i}\right)\right)_{i=0, \ldots, N}\right) & \geq A\left(-2^{1+\alpha} C_{0} A^{\alpha} e^{(1+\alpha) \lambda^{+}\left(x+r^{*}\right)}|E|^{1+\alpha}+e^{\lambda^{\prime} x} g\left(\lambda^{\prime}\right)\right) \\
& \geq A\left(-2^{1+\alpha} C_{0} A^{\alpha} e^{(1+\alpha) \lambda^{+} r^{*}}|E|^{1+\alpha}+g\left(\lambda^{\prime}\right)\right) e^{\lambda^{\prime} x}
\end{aligned}
$$

with $E=(1, \ldots, 1) \in \mathbb{R}^{N+1}$. Again, since $g\left(\lambda^{\prime}\right)>0$, then $\bar{\phi}$ is a super-solution of (5.73) for $A>0$ small enough.

Step 2.2: applying the comparison principle

Define, for $a>0$ large enough, the function $\tilde{\phi}(x)=\phi(x-a)$ such that

$$
\tilde{\phi} \leq \min \left(\beta_{0}, A e^{-\lambda^{+} r^{*}}\right) \quad \text { on } \quad(-\infty, 0]
$$


with $\beta_{0}$ given in assumption $(C)$. This is possible because we assume that $\phi(-\infty)=0$. Thus

$$
\tilde{\phi} \leq A e^{-\lambda^{+} r^{*}} \leq \bar{\phi} \quad \text { on } \quad\left[-r^{*}, 0\right] .
$$

Hence, applying the comparison principle result (Theorem 4.1, up to a shift of the functions), we deduce that

$$
\tilde{\phi} \leq \bar{\phi} \quad \text { on } \quad(-\infty, 0] .
$$

This implies that

$$
\frac{\phi(x)}{e^{\lambda^{+} x}} \leq 2 A e^{\lambda^{+} a} \text { for all } x \leq-a .
$$

Using the fact that $\phi \leq 1$, we get

$$
\frac{\phi(x)}{e^{\lambda^{+} x}} \leq k_{2} \text { for all } x \leq 0,
$$

where $k_{2}:=\max \left(2 A e^{\lambda^{+} a}, \max _{x \in[-a, 0]} \frac{\phi(x)}{e^{\lambda^{+} x}}\right)$.

We only prove Proposition $5.1 \mathrm{i}$ ) (the proof of Proposition $5.1 \mathrm{ii}$ ) being similar).

Proof of Proposition 5.1 i)

Let $\phi:[0,1] \rightarrow \mathbb{R}$ be a solution of (5.67) such that

$$
\phi(-\infty)=0 \quad \text { and } \quad \phi \geq \delta \quad \text { for some } \delta>0 .
$$

We recall, from Lemma 5.4, that

$$
0<k_{1} \leq \frac{\phi(x)}{e^{\lambda^{+} x}} \leq k_{2}<+\infty \text { for all } x \leq 0,
$$

where $\lambda^{+}$is the solution of (5.68).

\section{Step 1: Shifting and rescaling $\phi$}

For a sequence $x_{n} \rightarrow-\infty$ and for all $x \leq 0$, define the function $v_{n}$ as

$$
v_{n}\left(x-x_{n}\right):=\frac{\phi(x)}{e^{\lambda^{+} x}} .
$$

We have

$$
c \phi^{\prime}(x)=c e^{\lambda^{+} x}\left(v_{n}^{\prime}\left(x-x_{n}\right)+\lambda^{+} v_{n}\left(x-x_{n}\right)\right)=F\left(\left(v_{n}\left(x+r_{i}-x_{n}\right) e^{\lambda^{+}\left(x+r_{i}\right)}\right)_{i}\right)
$$

That is, for $y=x-x_{n}$,

$$
\begin{aligned}
c\left(v_{n}^{\prime}(y)+\lambda^{+} v_{n}(y)\right)= & e^{-\lambda^{+}\left(y+x_{n}\right)} F\left(\left(v_{n}\left(y+r_{i}\right) e^{\lambda^{+}\left(y+x_{n}+r_{i}\right)}\right)_{i}\right) \\
= & e^{-\lambda^{+}\left(y+x_{n}\right)}\left[F\left(\left(v_{n}\left(y+r_{i}\right) e^{\lambda^{+}\left(y+x_{n}+r_{i}\right)}\right)_{i}\right)-\nabla F(0) .\left(\left(v_{n}\left(y+r_{i}\right) e^{\lambda^{+}\left(y+x_{n}+r_{i}\right)}\right)_{i}\right)\right] \\
& \quad+\sum_{i=0}^{N} \frac{\partial F}{\partial X_{i}}(0) v_{n}\left(y+r_{i}\right) e^{\lambda^{+} r_{i}} .
\end{aligned}
$$

From assumption $(E+) i i)$, we then have

$$
c\left(v_{n}^{\prime}(y)+\lambda^{+} v_{n}(y)\right)=\sum_{i=0}^{N} \frac{\partial F}{\partial X_{i}}(0) v_{n}\left(y+r_{i}\right) e^{\lambda^{+} r_{i}}+O\left(e^{-\lambda^{+}\left(y+x_{n}\right)}\left|\left(v_{n}\left(y+r_{i}\right) e^{\lambda^{+}\left(y+x_{n}+r_{i}\right)}\right)_{i}\right|^{1+\alpha}\right)
$$


i.e,

$$
c\left(v_{n}^{\prime}(y)+\lambda^{+} v_{n}(y)\right)=\sum_{i=0}^{N} \frac{\partial F}{\partial X_{i}}(0) v_{n}\left(y+r_{i}\right) e^{\lambda^{+} r_{i}}+O\left(e^{\lambda^{+} \alpha\left(y+x_{n}\right)}\left|\left(v_{n}\left(y+r_{i}\right) e^{\lambda^{+} r_{i}}\right)_{i}\right|^{1+\alpha}\right)
$$

Step 2: Passing to the limit $n \rightarrow+\infty$

Because of (5.74), we have

$$
0<k_{1} \leq v_{n}(y) \leq k_{2}<+\infty \text { for } y \leq-x_{n}
$$

and for any compact set $K \subset \mathbb{R}$

$$
\left.e^{\lambda^{+} \alpha\left(y+x_{n}\right)}\left|\left(v_{n}\left(y+r_{i}\right) e^{\lambda^{+} r_{i}}\right)_{i}\right|^{1+\alpha} \rightarrow 0 \quad \text { as } n \rightarrow+\infty \quad \text { (because } x_{n} \rightarrow-\infty\right)
$$

uniformly in $y \in K$. As $c \neq 0$, we get from (5.76) that there exists some $C_{K}>0$ (independent on $n$ ) such that

$$
\left|v_{n}^{\prime}\right| \leq C_{K} \quad \text { on } \quad K
$$

Applying Ascoli's theorem, there exists a subsequence $v_{n^{\prime}}$ such that

$$
v_{n^{\prime}} \longrightarrow v_{\infty} \quad \text { locally uniformly on } \mathbb{R} \text {. }
$$

Moreover $v_{\infty}$ satisfies

$$
c\left(v_{\infty}^{\prime}(y)+\lambda^{+} v_{\infty}(y)\right)=\sum_{i=0}^{N} \frac{\partial F}{\partial X_{i}}(0) v_{\infty}\left(y+r_{i}\right) e^{\lambda^{+} r_{i}}
$$

and (using (5.77))

$$
k_{1} \leq v_{\infty} \leq k_{2} \quad \text { on } \quad \mathbb{R}
$$

\section{Step 3: Applying Fourier transform}

Applying Fourier transform to (5.78), implies that

$$
\hat{v}_{\infty}(\xi) G(\xi)=0
$$

where $G(\xi)=c\left(i \xi+\lambda^{+}\right)-\sum_{j=0}^{N} \frac{\partial F}{\partial X_{j}}(0, \ldots, 0) e^{\lambda^{+} r_{j}} e^{i \xi r_{j}}$.

Step 3.1: $G(\xi)=0 \Longleftrightarrow \xi=0$

Clearly, if $\xi=0$ then $G(\xi)=0$ (because $\lambda^{+}$solves (5.68)).

Assume that $G(\xi)=0$ with $\xi \in \mathbb{R}$. Hence

$$
c \lambda^{+}=\sum_{j=0}^{N} \frac{\partial F}{\partial X_{j}}(0, \ldots, 0) e^{\lambda^{+} r_{j}} \cos \left(\xi r_{j}\right)
$$

and

$$
c \xi=\sum_{j=0}^{N} \frac{\partial F}{\partial X_{j}}(0, \ldots, 0) e^{\lambda^{+} r_{j}} \sin \left(\xi r_{j}\right)
$$


Using the fact that $\frac{\partial F}{\partial X_{j}}(0) \geq 0$ for $j \neq 0$, we deduce from (5.68) and (5.80) that for all $j \in\{1, \ldots, N\}$, we have

$$
\left\{\begin{array}{l}
\frac{\partial F}{\partial X_{j}}(0, \ldots, 0)=0 \\
\text { or } \\
\xi r_{j}=0 \quad \bmod (2 \pi) \text { and } \frac{\partial F}{\partial X_{j}}(0)>0 .
\end{array}\right.
$$

Substituting (5.82) in (5.81), taking into consideration that $r_{0}=0$, implies that $c \xi=0$ and thus $\xi=0$, because $c \neq 0$.

Step 3.2: $v_{\infty}=$ const

From step 3.1, we deduce that $\operatorname{supp}\{\hat{v}\} \subset\{0\}$. Therefore,

$$
\hat{v}(0)=\sum_{\text {finite }} c_{k} \delta_{0}^{(k)}
$$

Inverse Fourier transform implies that $v_{\infty}$ is a polynomial. But $v_{\infty}$ is bounded (see (5.79)), hence

$$
v_{\infty}=\text { const }:=A \text {. }
$$

Consequently,

$$
\frac{\phi\left(x+x_{n^{\prime}}\right)}{e^{\lambda^{+}\left(x+x_{n^{\prime}}\right)}}=v_{n^{\prime}}(x) \rightarrow A .
$$

\section{Uniqueness of the profile and proof of Theorem 1.5}

We prove, in this section, the uniqueness of the profile (under the assumption $(D)$ or $(E)$ ). Under Assumption $(D)$ we will use a Strong Maximum Principle, while under assumption $(E)$ we will need the asymptotics joint to a Half Strong Maximum Principle (just on the half-line, see Lemma 6.1). We show, in a first subsection, three different kinds of Strong Maximum Principle satisfied by (1.14) when $c \neq 0$. In a second subsection, we prove the uniqueness of the profile and Theorem 1.5.

\subsection{Different kinds of Strong Maximum Principle}

Here, we prove three different kinds of Strong Maximum Principle for $(1.14)$ when $c \neq 0$. We also add a technical lemma (Lemma 6.5) that allow us to compare two different solutions on $\mathbb{R}$ with at least one contact point.

We prove the Strong Maximum Principle (Lemma 6.1, 6.3 and 6.4) for $c>0$. However, when $c<0$, the corresponding results can be deduced from the case $c>0$ using the transformation of Lemma 4.4. 


\section{Lemma 6.1 (Half Strong Maximum Principle)}

Let $F:[0,1]^{N+1} \rightarrow \mathbb{R}$ satisfying assumption $(A)$ and let $\phi_{1}, \phi_{2}: \mathbb{R} \rightarrow[0,1]$ be respectively a viscosity sub and a super-solution of (2.21). Assume that

$$
\left\{\begin{array}{l}
\phi_{2} \geq \phi_{1} \text { on } \mathbb{R} \\
\phi_{2}(0)=\phi_{1}(0)
\end{array}\right.
$$

If $c>0$ (resp. $c<0)$, then

$$
\left.\phi_{1}=\phi_{2} \quad \text { for all } x \leq 0 \quad \text { (resp. } x \geq 0\right) .
$$

\section{Proof of Lemma 6.1}

Assume that $c>0$ and let $w(x):=\phi_{2}(x)-\phi_{1}(x)$. Since $\phi_{2}$ is a super-solution and $\phi_{1}$ is a sub-solution of (2.21), then using the Doubling of variable method we show that $w$ is a viscosity super-solution of

$$
c w^{\prime}(x) \geq F\left(\left(\phi_{2}\left(x+r_{i}\right)\right)_{i=0, \ldots, N}\right)-F\left(\left(\phi_{1}\left(x+r_{i}\right)\right)_{i=0, \ldots, N}\right) \quad \text { on } \quad \mathbb{R} .
$$

But $F$ is non-decreasing w.r.t. $X_{i}$ for all $i \neq 0$, thus we get

$$
c w^{\prime}(x) \geq F\left(\phi_{1}(x)+w(x),\left(\phi_{1}\left(x+r_{i}\right)\right)_{i=1, \ldots, N}\right)-F\left(\left(\phi_{1}\left(x+r_{i}\right)\right)_{i=0, \ldots, N}\right) .
$$

Now, since $F$ is globally Lipschitz, then

$$
w^{\prime}(x) \geq \frac{-L}{c} w(x)
$$

with $L$ is the Lipschitz constant of $F$.

Notice that $y(x)=w\left(x_{0}\right) e^{\frac{-L}{c}\left(x-x_{0}\right)}$ satisfies the equality in inequality (6.83) for any $x_{0} \in \mathbb{R}$. As $y\left(x_{0}\right)=w\left(x_{0}\right)$, then using the comparison principle for the "ode" (6.83), we deduce that

$$
w(x) \geq w\left(x_{0}\right) e^{\frac{-L}{c}\left(x-x_{0}\right)} \quad \text { for all } \quad x \geq x_{0} .
$$

If $w\left(x_{0}\right)>0$, hence $w(x)>0$ for all $x \geq x_{0}$. This implies that

$$
\phi_{2}>\phi_{1} \text { for all } x \geq x_{0} .
$$

Finally, since $\phi_{2}(0)=\phi_{1}(0)$, then we deduce that

$$
\phi_{2}=\phi_{1} \text { for all } x \leq 0,
$$

(otherwise, if there is $x_{1}<0$ such that $\phi_{2}\left(x_{1}\right)>\phi_{1}\left(x_{1}\right)$, then from the above argument, we deduce that $\phi_{2}(0)>\phi_{1}(0)$, a contradiction).

Lemma 6.2 (Strong Maximum Principle under $(D \pm)$ ii))

Let $F:[0,1]^{N+1} \rightarrow \mathbb{R}$ satisfying $(A)$. Let $\phi_{1}, \phi_{2}: \mathbb{R} \rightarrow[0,1]$ be respectively a viscosity sub and super-solution of (2.21) such that

$$
\phi_{2} \geq \phi_{1} \quad \text { on } \quad \mathbb{R} \quad \text { and } \quad \phi_{2}(0)=\phi_{1}(0)
$$


a) If $F$ is increasing w.r.t. $X_{i_{0}}$ for certain $i_{0} \neq 0$ then

$$
\phi_{2}\left(k r_{i_{0}}\right)=\phi_{1}\left(k r_{i_{0}}\right) \quad \text { for all } k \in \mathbb{N} \text {. }
$$

b) If we assume moreover that $F$ satisfies $(D+)$ ii) if $c>0$, or $(D-)$ ii) if $c<0$, then

$$
\phi_{1}(x)=\phi_{2}(x) \quad \text { for all } x \in \mathbb{R} \text {. }
$$

\section{Proof of Lemma 6.2}

a) Assume for simplicity that $i_{0}=1$. Let $\phi_{1}, \phi_{2}$ be respectively a viscosity sub and a viscosity super-solution of (2.21). Then using the Doubling of variable method, we can show that the function $w=\phi_{2}-\phi_{1}$ satisfies

$$
c w^{\prime}(x) \geq F\left(\left(\phi_{2}\left(x+r_{i}\right)\right)_{i=0, \ldots, N}\right)-F\left(\left(\phi_{1}\left(x+r_{i}\right)\right)_{i=0, \ldots, N}\right) \quad \text { on } \quad \mathbb{R}
$$

in the viscosity sense. As $w$ is a viscosity super-solution of (6.85), $w(0)=0$ and $w \geq 0$ on $\mathbb{R}$, we deduce that

$$
0 \geq F\left(\left(\phi_{2}\left(r_{i}\right)\right)_{i=0, \ldots, N}\right)-F\left(\left(\phi_{1}\left(r_{i}\right)\right)_{i=0, \ldots, N}\right) \text { at } \quad x=0 .
$$

Thus using the fact that $\phi_{2}(0)=\phi_{1}(0)$ and that $F$ is monotone w.r.t. $X_{i}$ for all $i \neq 0$, we get

$$
F\left(\left(\phi_{2}\left(r_{i}\right)\right)_{i=0, \ldots, N}\right)=F\left(\left(\phi_{1}\left(r_{i}\right)\right)_{i=0, \ldots, N}\right) .
$$

Next, since $F$ is increasing w.r.t. $X_{1}$, we deduce that

$$
\phi_{2}=\phi_{1} \quad \text { at } \quad x=r_{1}
$$

(otherwise, $F\left(\left(\phi_{2}\left(r_{i}\right)\right)_{i=0, \ldots, N}\right)>F\left(\left(\phi_{1}\left(r_{i}\right)\right)_{i=0, \ldots, N}\right)$, because $F$ is non-decreasing w.r.t. $X_{i}$ for $i \neq 0,1$ and increasing w.r.t. $\left.X_{1}\right)$. Therefore, upon repeating the above argument for $x=r_{1}$, we show that

$$
\phi_{2}\left(k r_{1}\right)=\phi_{1}\left(k r_{1}\right) \quad \text { for all } \quad k \in \mathbb{N} \text {. }
$$

b) Assume that $c>0$ and that $F$ satisfies $(D+) i i$ ) (the other case being similar). By contradiction, suppose that there exists $x \in \mathbb{R}$ such that $\phi_{1}(x)<\phi_{2}(x)$. Let $k \in \mathbb{N}$ big enough such that $k r_{i_{+}}>x$. Then, using Lemma 6.1 (up to shift the functions), and the fact that $\phi_{1}\left(k r_{i_{+}}\right)=\phi_{2}\left(k r_{i_{+}}\right)$, we get that $\phi_{1}(x)=\phi_{2}(x)$, which is a contradiction.

\section{Lemma 6.3 (Comparison principle, under $(D \pm) i)$ )}

Assume that $c>0$ (resp. $c<0)$ and let $F$ satisfying $(A)$ and $(D+) i)($ resp. $(D-) i))$. Let $\phi_{1}, \phi_{2}$ be respectively a viscosity sub and a viscosity super-solution of (2.21). Assume that $\phi_{1}(0)=\phi_{2}(0)$ and

$$
\phi_{1} \leq \phi_{2} \quad \text { on } \quad\left[-r^{*}, 0\right] \quad\left(r e s p \text {. on }\left[0, r^{*}\right]\right) \text {, }
$$

then

$$
\phi_{1}(x) \leq \phi_{2}(x) \quad \text { for all } \quad x \geq-r^{*} \quad\left(\text { resp. } x \leq r^{*}\right)
$$




\section{Proof of Lemma 6.3}

Assume that $c>0$ (the case $c<0$ being similar). If $r^{*}=0$, then the result follows from the comparison principle for ODEs.

Let us assume that $r^{*}>0$. Since $\phi_{1} \leq \phi_{2}$ on $\left[-r^{*}, 0\right]$ and $r_{i}<0$ for all $i \neq 0$ (see assumption $(D+) i)$ ), then for all $x \in\left[0, \min _{i \neq 0}\left(-r_{i}\right)\right]$, the function $w(x):=\phi_{1}(x)-\phi_{2}(x)$ satisfies (in the viscosity sense)

$$
\begin{aligned}
c w^{\prime}(x) & \leq F\left(\left(\phi_{1}\left(x+r_{i}\right)\right)_{i=0, \ldots, N}\right)-F\left(\left(\phi_{2}\left(x+r_{i}\right)\right)_{i=0, \ldots, N}\right) \\
& \leq F\left(w(x)+\phi_{2}(x),\left(\phi_{2}\left(x+r_{i}\right)\right)_{i \neq 0}\right)-F\left(\left(\phi_{2}\left(x+r_{i}\right)\right)_{i=0, \ldots, N}\right) \\
& \leq L|w(x)| \quad \text { (because } F \text { is } L \text {-Lipschitz }) .
\end{aligned}
$$

where we have used in the second line the fact that $\phi_{1}\left(x+r_{i}\right) \leq \phi_{2}\left(x+r_{i}\right)$ for $i \neq 0$, because $-r^{*} \leq x+r_{i} \leq 0$ for all $i \neq 0$. But $w(0)=0$ and $y \equiv 0$ is a solution of $c w^{\prime}=L|w|$, then using the comparison principle of the "ode," we deduce that

$$
w \leq 0 \text { for all } x \in\left[0, \min _{i \neq 0}\left(-r_{i}\right)\right] .
$$

This implies that

$$
\phi_{1} \leq \phi_{2} \quad \text { for all } \quad x \in\left[0, \min _{i \neq 0}\left(-r_{i}\right)\right] .
$$

Finally, the result of this lemma $\left(\phi_{1} \leq \phi_{2}\right.$ for all $\left.x \geq-r^{*}\right)$ follows by repeating the above argument several times, each on the new extended interval.

\section{Lemma 6.4 (Strong Maximum principle under $(D \pm) i$ ))}

Assume $c>0$ (resp. $c<0$ ) and let $F$ satisfying $(A)$ and $(D+) i)($ resp. $(D-) i)$ ). Let $\phi_{1}, \phi_{2}$ be two solutions of (2.21) such that

$$
\phi_{1}(0)=\phi_{2}(0) \quad \text { and } \quad \phi_{1} \leq \phi_{2} \quad \text { on } \quad \mathbb{R} .
$$

Then

$$
\phi_{1}(x)=\phi_{2}(x) \quad \text { for all } \quad x \in \mathbb{R} .
$$

\section{Proof of Lemma 6.4}

Let $c>0$ (the case $c<0$ is deduced from the case $c>0$ using Lemma 4.4). Using Lemma 6.1 , we deduce that

$$
\phi_{1}=\phi_{2} \text { for all } x \leq 0 .
$$

Thus, it is sufficient to prove that $\phi_{1} \geq \phi_{2}$ for all $x \geq 0$ (because $\phi_{1} \leq \phi_{2}$ for $x \geq 0$ ). We have,

$$
\phi_{1}(0)=\phi_{2}(0) \quad \text { and } \quad \phi_{1} \geq \phi_{2} \quad \text { on } \quad\left[-r^{*}, 0\right] \quad\left(\text { since } \phi_{1}=\phi_{2} \quad \forall x \leq 0\right),
$$

and $\phi_{2}, \phi_{1}$ are respectively a viscosity sub and super-solution of (2.21). Hence using the comparison principle (Lemma 6.3), we deduce that

$$
\phi_{1} \geq \phi_{2} \text { for all } x \geq-r^{*} .
$$

Therefore, $\phi_{1}(x)=\phi_{2}(x)$ for all $x \in \mathbb{R}$. 


\section{Lemma 6.5 (Ordering two solutions of (1.14) up to translation)}

Assume that $c \neq 0$ and let $F:[0,1]^{N+1} \rightarrow \mathbb{R}$ satisfying $(A)$ and $(C)$. Let $\phi_{1}$ and $\phi_{2}$ be two solutions of (1.14). There exists a shift $a^{*} \in \mathbb{R}$ and some $x_{0} \in\left[-r^{*}, r^{*}\right]$ such that $\phi_{2}^{a^{*}}(x):=\phi_{2}\left(x+a^{*}\right)$ and $\phi_{1}$ satisfy

$$
\left\{\begin{array}{l}
\phi_{2}^{a^{*}} \geq \phi_{1} \quad \text { on } \quad \mathbb{R} \\
\phi_{2}^{a^{*}}\left(x_{0}\right)=\phi_{1}\left(x_{0}\right) .
\end{array}\right.
$$

\section{Proof of Lemma 6.5}

The idea of the proof is to translate $\phi_{2}$ and then to compare the translation with $\phi_{1}$.

\section{Step 1: Family of solutions above $\phi_{1}$}

For $a \in \mathbb{R}$, let us define

$$
\phi_{2}^{a}(x):=\phi_{2}(x+a) .
$$

For some $a>0$ large enough, (because of the conditions at $\pm \infty$ in (1.14)), we have

$$
\phi_{2}^{\bar{a}} \geq \phi_{1} \quad \text { on }\left[-r^{*}, r^{*}\right] \text { for all } \bar{a} \geq a,
$$

and then using the comparison principle (Theorem 4.1 and Corollary 4.2), we deduce that for all $\bar{a} \geq a$, we have

$$
\phi_{2}^{\bar{a}} \geq \phi_{1} \quad \text { on } \quad \mathbb{R} \text {. }
$$

Step 2: There exists $a^{*}$ such that $\phi_{2}^{a^{*}}$ and $\phi_{1}$ touch at $x_{0} \in\left[-r^{*}, r^{*}\right]$ Let

$$
a^{*}=\inf \left\{a \in \mathbb{R}, \quad \phi_{2}^{\bar{a}} \geq \phi_{1} \quad \text { on } \quad \mathbb{R} \quad \text { for all } \bar{a} \geq a\right\} .
$$

Recall that $c \neq 0$ and then $\phi_{i} \in C^{1}(\mathbb{R})$ for $i=1,2$.

Assume by contradiction that

$$
\inf _{\left[-r^{*}, r^{*}\right]}\left(\phi_{2}^{a^{*}}-\phi_{1}\right) \geq \delta>0
$$

Then for all $0 \leq \varepsilon \leq \varepsilon_{0}$ with $\varepsilon_{0}$ small enough, we have

$$
\phi_{2}^{a^{*}-\varepsilon}-\phi_{1} \geq 0 \quad \text { on } \quad\left[-r^{*}, r^{*}\right] .
$$

Applying the comparison principle (Theorem 4.1 and Corollary 4.2), we get

$$
\phi_{2}^{a^{*}-\varepsilon}-\phi_{1} \geq 0 \quad \text { on } \quad \mathbb{R},
$$

which is a contradiction with the definition of $a^{*}$. Thus

$$
\inf _{\left[-r^{*}, r^{*}\right]} \phi_{2}^{a^{*}}-\phi_{1}=0
$$

Hence, there exists $x_{0} \in\left[-r^{*}, r^{*}\right]$ such that

$$
\phi_{2}^{a^{*}}=\phi_{1} \quad \text { at } \quad x_{0}
$$

knowing that $\phi_{2}^{a^{*}}(x) \geq \phi_{1}(x)$ for all $x \in \mathbb{R}$. 


\subsection{Proof of Theorem $1.5(b)$}

We devote this subsection for the proof of the uniqueness of the profile which is done in several lemmas. The proof of Theorem 1.5 is given at the end of this subsection. All the proofs are made in the case $c>0$ since the case $c<0$ is similar (or is deduced using Lemma 4.4).

Lemma 6.6 (Uniqueness of the profile, under $(E+)$ )

Assume that $c>0$ and let $F$ satisfying $(A),(C)$ and $(E+)$. Let $\phi: \mathbb{R} \rightarrow[0,1]$ be a solution of (1.14), then $\phi$ is unique up to space translation. Moreover $\phi$ is non-decreasing.

\section{Proof of Lemma 6.6}

Assume that $c>0$ and let $\phi_{1}, \phi_{2}: \mathbb{R} \rightarrow[0,1]$ be two solutions of (1.14). The goal of the proof is to show that there exists a translation $\phi_{2}^{a^{*}}$ of $\phi_{2}$ such that $\phi_{2}^{a^{*}}=\phi_{1}$. To simplify the notation we denote $r_{i_{+}}$(introduced in $(E+)$ ) by $r_{1}$.

\section{Step 1: Constructing a translation and applying Lemma 6.1}

Using Lemma 6.5, there exists $a^{*} \in \mathbb{R}$ such that the translation $\phi_{2}^{a^{*}}$ of $\phi_{2}$ satisfies:

$$
\left\{\begin{array}{l}
\phi_{2}^{a^{*}} \geq \phi_{1} \quad \text { on } \quad \mathbb{R} \\
\phi_{2}^{a^{*}}\left(x_{0}\right)=\phi_{1}\left(x_{0}\right) .
\end{array}\right.
$$

Since $c>0$, then applying Lemma 6.1, we deduce that

$$
\phi_{2}^{a^{*}}=\phi_{1} \quad \text { for all } \quad x \leq x_{0} .
$$

Step 2: Asymptotics of $\phi_{1}$ and $\phi_{2}^{a^{*}}$

Using Lemma 5.2 and Proposition 5.1, we get that there exists a subsequence $\left(n^{\prime}\right)$ of $(n)_{n \in \mathbb{N}}$ (because $x_{0}-n r_{1} \rightarrow-\infty$ as $n \rightarrow+\infty$ ) and two constants $A_{1}, A_{2}>0$ such that

$$
\begin{array}{lll}
\frac{\phi_{2}^{a^{*}}\left(x_{0}-n^{\prime} r_{1}+x\right)}{e^{\lambda^{+}\left(x_{0}-n^{\prime} r_{1}+x\right)}} \rightarrow A_{1} \quad \text { locally uniformly on } & \mathbb{R} . \\
\frac{\phi_{1}\left(x_{0}-n^{\prime} r_{1}+x\right)}{e^{\lambda^{+}\left(x_{0}-n^{\prime} r_{1}+x\right)}} \rightarrow A_{2} & \text { locally uniformly on } & \mathbb{R} .
\end{array}
$$

Using equation (6.87), we deduce that $A_{1}=A_{2}:=A$.

Step 3: Exchange $\phi_{1}$ and $\phi_{2}$

Applying Lemma 6.5, upon exchanging $\phi_{1}$ and $\phi_{2}$, we deduce that there exists $b^{*} \geq 0$ and $y_{0}$ such that

$$
\left\{\begin{array}{l}
\phi_{1}^{b^{*}}(x):=\phi_{1}\left(x+b^{*}\right) \geq \phi_{2} \quad \text { on } \quad \mathbb{R} \\
\phi_{1}^{b^{*}}\left(y_{0}\right)=\phi_{2}\left(y_{0}\right) .
\end{array}\right.
$$

Moreover, from Lemma 6.1, we get

$$
\phi_{1}^{b^{*}}(x)=\phi_{2} \quad \text { for all } \quad x \leq y_{0} \quad(\text { since } c>0) .
$$

Now, using and Lemma 5.2 and Proposition 5.1 and since $y_{0}-n^{\prime} r_{1} \rightarrow-\infty$ as $n^{\prime} \rightarrow+\infty$, we get the existence of a subsequence of $\left(n^{\prime}\right)$ (still denoted by $\left(n^{\prime}\right)$ ) such that

$$
\frac{\phi_{1}^{b^{*}}\left(y_{0}-n^{\prime} r_{1}+x\right)}{e^{\lambda^{+}\left(y_{0}-n^{\prime} r_{1}+x\right)}}, \quad \frac{\phi_{2}\left(y_{0}-n^{\prime} r_{1}+x\right)}{e^{\lambda^{+}\left(y_{0}-n^{\prime} r_{1}+x\right)}} \rightarrow B \quad \text { locally uniformly on } \mathbb{R} .
$$


Step 4: Conclusion, $\phi_{1}=\phi_{2}^{a^{*}}$

For any fixed $x \in \mathbb{R}$, we have

$$
\begin{gathered}
\frac{\phi_{2}\left(x_{0}+a^{*}-n^{\prime} r_{1}+x\right)}{e^{\lambda^{+}\left(x_{0}-n^{\prime} r_{1}+x\right)}} \rightarrow A, \\
\frac{\phi_{1}\left(x_{0}-n^{\prime} r_{1}+x\right)}{e^{\lambda^{+}\left(x_{0}-n^{\prime} r_{1}+x\right)}} \rightarrow A, \\
\frac{\phi_{1}\left(y_{0}+b^{*}-n^{\prime} r_{1}+x\right)}{e^{\lambda^{+}\left(y_{0}-n^{\prime} r_{1}+x\right)}} \rightarrow B
\end{gathered}
$$

and

$$
\frac{\phi_{2}\left(y_{0}-n^{\prime} r_{1}+x\right)}{e^{\lambda^{+}\left(y_{0}-n^{\prime} r_{1}+x\right)}} \rightarrow B
$$

For $x=y_{0}+b^{*}$, equation (6.91) implies that

$$
\frac{\phi_{1}\left(x_{0}-n^{\prime} r_{1}+y_{0}+b^{*}\right)}{e^{\lambda^{+}\left(x_{0}-n^{\prime} r_{1}+y_{0}\right)}} \rightarrow A e^{\lambda^{+} b^{*}} .
$$

Also, equation (6.92) with $x=x_{0}$ implies that

$$
\frac{\phi_{1}\left(x_{0}-n^{\prime} r_{1}+y_{0}+b^{*}\right)}{e^{\lambda^{+}\left(x_{0}-n^{\prime} r_{1}+y_{0}\right)}} \rightarrow B
$$

thus

$$
A e^{\lambda^{+} b^{*}}=B .
$$

Similarly, if we substitute $x=y_{0}$ in (6.90) and $x=x_{0}+a^{*}$ in (6.93), we show that

$$
A=B e^{\lambda^{+} a^{*}} .
$$

Therefore,

$$
a^{*}=-b^{*}
$$

But

$$
\phi_{2}^{a^{*}}(x)=\phi_{2}\left(x+a^{*}\right) \geq \phi_{1}(x)
$$

and

$$
\phi_{1}^{b^{*}}(x)=\phi_{1}\left(x+b^{*}\right)=\phi_{1}\left(x-a^{*}\right) \geq \phi_{2}(x),
$$

hence we get

$$
\phi_{2}\left(x+a^{*}\right)=\phi_{1}(x) .
$$

Moreover $\phi_{2}(x+a) \geq \phi_{1}(x)$ for all $a \geq a^{*}$, which shows that the profile is nondecreasing.

Lemma 6.7 (Uniqueness of the profile, under $(D+) i)$ or $i i)$ )

Assume that $c>0$ and let $F$ satisfying $(A)$ and $(C)$. Let $\phi: \mathbb{R} \rightarrow[0,1]$ be a solution of (1.14). If, in addition, $F$ satisfies $(D+)(i)$ or $i i)$, then $\phi$ is unique up to space translation. 


\section{Proof of Lemma 6.7}

The proof follows from Lemma 6.5 and the Strong Maximum Principle (Lemma 6.4 or Lemma $6.2)$.

\section{Lemma 6.8 (Monotonicity of the profile)}

Assume that $c>0$ (resp. $c<0$ ) and let $F:[0,1]^{N+1} \rightarrow \mathbb{R}$ satisfying $(A)$ and $(C)$. Let $\phi: \mathbb{R} \rightarrow[0,1]$ be a solution of (1.14). If $F$ satisfies $(D+) i)$ or $i i)$ or $(E+)$ (resp. $(D-) i)$ or $i$ i) or $(E-))$, then $\phi^{\prime}>0$ on $\mathbb{R}$.

\section{Proof of Lemma 6.8}

Assume that $c>0$ (the proof when $c<0$ being similar) and let $\phi$ be a solution of (1.14).

\section{Step 1: $\phi$ is non-decreasing}

The goal is to show that $\phi(x+a) \geq \phi(x)$ for all $a \geq 0$. As in the proof of Lemma 6.5 , we deduce that for $a \geq 0$ large enough and for all $\bar{a} \geq a$, we have

$$
\phi^{\bar{a}}(x):=\phi(x+\bar{a}) \geq \phi(x) \quad \text { on } \quad\left[-r^{*}, r^{*}\right] .
$$

Thus using the comparison principle (Theorem 4.1 and Corollary 4.2), we deduce that for all $\bar{a} \geq a$, we have

$$
\phi^{\bar{a}}(x) \geq \phi(x) \quad \text { on } \quad \mathbb{R} .
$$

Set

$$
a^{*}=\inf \left\{a \geq 0, \phi^{\bar{a}}(x) \geq \phi(x) \text { on } \mathbb{R} \text { for all } \bar{a} \geq a\right\},
$$

we want to prove that $a^{*}=0$. By definition of $a^{*}$, there exists some $x_{0}$ such that

$$
\left\{\begin{array}{l}
\phi^{a^{*}} \geq \phi \quad \text { on } \quad \mathbb{R} \\
\phi^{a^{*}}\left(x_{0}\right)=\phi\left(x_{0}\right) .
\end{array}\right.
$$

Case 1: $F$ satisfies $(E+)$

From Lemma 6.6, $\phi$ is nondecreasing and then $a^{*}=0$.

Case 2: $F$ satisfies $(D+) i)$ or $i i)$

Using (6.94) and the Strong Maximum Principle (Lemma 6.2 or Lemma 6.4), we get that $\phi^{a^{*}}=\phi$, i.e., $\phi$ is periodic of period $a^{*}$. But $\phi(-\infty)=0$ and $\phi(+\infty)=1$, thus $a^{*}=0$.

\section{Step 2: $\phi$ is increasing}

Let $a>0$, we want to show that $\phi(x+a)>\phi(x)$. From Step 1, we have $\phi(x+a) \geq \phi(x)$. Assume that there exists $x_{0}$ such that

$$
\phi\left(x_{0}+a\right)=\phi\left(x_{0}\right)
$$

Repeating the same argument, as in Step 1, under $(D+) i)$ or $i i)$ or $(E+)$, we prove that $a=0$, which is a contradiction. Thus

$$
\phi(x+a)>\phi(x) \quad \text { on } \quad \mathbb{R} \text { for any } a>0 .
$$


Step 3: $\phi^{\prime}>0$

For $a>0$, we define

$$
w_{a}(x)=\frac{\phi(x+a)-\phi(x)}{a} .
$$

Using the same arguments as in the proof of Lemma 6.1 (see (6.84)), we get that for all $x_{0} \in \mathbb{R}$

$$
w_{a}(x) \geq w_{a}\left(x_{0}\right) e^{\frac{-L}{c}\left(x-x_{0}\right)} \quad \text { for all } \quad x \geq x_{0} .
$$

Passing to the limit $a \rightarrow 0$, we get that

$$
\phi^{\prime}(x) \geq \phi^{\prime}\left(x_{0}\right) e^{\frac{-L}{c}\left(x-x_{0}\right)} \geq 0 \text { for all } x \geq x_{0} .
$$

By contradiction, assume that there exists $x_{1}$ such that $\phi^{\prime}\left(x_{1}\right)=0$. This implies that

$$
\phi^{\prime}(x)=0 \text { for all } x \leq x_{1} .
$$

Indeed, if there exists $x_{0}<x_{1}$ such that $\phi^{\prime}\left(x_{0}\right)>0$, then (6.95) implies that

$$
\phi^{\prime}\left(x_{1}\right) \geq \phi^{\prime}\left(x_{0}\right) e^{\frac{-L}{c}\left(x_{1}-x_{0}\right)}>0,
$$

which is a contradiction.

But $\phi$ is increasing so (6.96) is a contradiction and so $\phi^{\prime}>0$.

\section{Proof of Theorem 1.5}

(a) Uniqueness of the velocity

The proof of the uniqueness of the velocity is follows from Proposition 4.5 in Section 4 .

\section{(b) Uniqueness of the profile and strict monotonicity}

The uniqueness and the strict monotonicity of the solution when $c>0$ is done in Lemma 6.6, 6.7 and Lemma 6.8. However the case $c<0$ is a consequence of Lemma 4.4 and the previous results.

\section{Appendix A Construction of a monotone Lipschitz continuous periodic extension of $F$}

We devote the Appendix A for the proof of Lemma 2.1. To this end, we need to start with two useful results about the orthogonal projection. For any convex set $K$ in $\mathbb{R}^{d}$ and for any $y \in \mathbb{R}^{d}$, we call

$$
\operatorname{Proj}_{\left.\right|_{K}}(y)
$$

the orthogonal projection of $y$ on $K$.

Lemma A.1 (Characterization of the orthogonal projection) Let $N \geq 1$ and $y=\left(y_{1}, \ldots, y_{N}\right) \in \mathbb{R}^{N}$. Then

$$
\operatorname{Proj}_{[0,1]}(y)=\left(\left(\operatorname{Proj}_{\left.\right|_{[0,1]}}\left(y_{i}\right)\right)_{i=1, \ldots, N}\right) .
$$




\section{Proof of Lemma A.1}

Let $y=\left(y_{1}, \ldots, y_{N}\right) \in \mathbb{R}^{N}$ and set $y_{i}^{0}=\operatorname{Proj}_{\mid[0,1]}\left(y_{i}\right)$. By definition of the orthogonal projection, we have

$$
\left(y_{i}-y_{i}^{0}, \bar{y}_{i}-y_{i}^{0}\right) \leq 0 \quad \forall \bar{y}_{i} \in[0,1]
$$

This implies that

$$
\left(y-y^{0}, \bar{y}-y^{0}\right) \leq 0 \quad \forall \bar{y}=\left(\bar{y}_{1}, \ldots, \bar{y}_{N}\right) \in[0,1]^{N},
$$

with $y^{0}=\left(y_{1}^{0}, \ldots, y_{N}^{0}\right)$. But (1.97) is a characterization of the orthogonal projection of $y$ on $[0,1]^{N}$, thus

$$
y^{0}=\operatorname{Proj}_{[0,1]^{N}}(y) \text {. }
$$

Using the above lemma, one can easily check the following consequences:

\section{Corollary A.2 (Ordering and a kind of linearity)}

Let $y=\left(y_{1}, \ldots, y_{N}\right), z=\left(z_{1}, \ldots, z_{N}\right) \in \mathbb{R}^{N}$ and set $e=(1, \ldots, 1) \in \mathbb{R}^{N}$. Assume that

$$
y \geq z
$$

in the sense that $y_{i} \geq z_{i}$ for all $i \in\{1, \ldots, N\}$. Let $Q_{0}=[0,1]^{N}$, then

\section{i) Order preservation}

We have

$$
\operatorname{Proj}_{Q_{0}}(y) \geq \operatorname{Proj}_{\left.\right|_{Q_{0}}}(z)
$$

\section{ii) "Linearity"}

We have

$$
\operatorname{Proj}_{\left.\right|_{0}}(y+e)=\operatorname{Proj}_{\left.\right|_{Q_{0}-e}}(y)+e,
$$

where $Q_{0}-e=[-1,0]^{N}$.

After these preliminary results, we now go back to the proof of Lemma 2.1.

\section{Proof of Lemma 2.1.}

The proof is splitted into two main steps. In the first step (the main part of the proof), we construct the extension $\tilde{F}$ of $F$ on $[0,1] \times \mathbb{R}^{N}$. In the second step, we extend $\tilde{F}$ on the whole $\mathbb{R} \times \mathbb{R}^{N}$. The function $\tilde{F}$ that we want to construct must satisfy

$$
\begin{cases}\tilde{F}_{\left.\right|_{Q}}=F & \text { for } \quad Q:=[0,1]^{N+1} \\ \tilde{F}(X+E)=\tilde{F}(X) & \text { with } E=(1, \ldots, 1) \in \mathbb{R}^{N+1} .\end{cases}
$$

This implies that for any $y \in Q_{0}=[0,1]^{N}$ and $e=(1, \ldots, 1) \in \mathbb{R}^{N}$, we have (see Figure 1 )

$$
\left\{\begin{array}{l}
\tilde{F}(1, y+e)=\tilde{F}(0, y)=F(0, y) \\
\tilde{F}(0, y-e)=\tilde{F}(1, y)=F(1, y) .
\end{array}\right.
$$

Step 1: extension on $[0,1] \times \mathbb{R}^{N}$

Recall that $Q_{0}=[0,1]^{N}, e=(1, \ldots, 1) \in \mathbb{R}^{N}$ then set

$$
Q_{-1}:=Q_{0}-e \text { and } \quad Q_{1}:=Q_{0}+e .
$$


We first define the auxiliary functions $G_{\alpha}$ on $[0,1] \times Q_{\alpha}$ for $\alpha=-1,0,1$. For $y=\left(y_{1}, \ldots, y_{N}\right) \in \mathbb{R}^{N}$, we set

$$
\mid \begin{array}{lll}
G_{0}(x, y)=F(x, y) & \text { for all } & (x, y) \in[0,1] \times Q_{0} \\
G_{-1}(x, y)=F(1, y+e)-F(1, e) & \text { for all } & (x, y) \in[0,1] \times Q_{-1} \\
G_{1}(x, y)=F(0, y-e)-F(0,0) & \text { for all } & (x, y) \in[0,1] \times Q_{1}
\end{array}
$$

By construction and using (assumption $(A)$ ), we notice that $G_{\alpha}$ is Lipschitz continuous and non-decreasing w.r.t. $y_{i}$ for all $i \in\{1, \ldots, N\}$ on $[0,1] \times Q_{\alpha}$, for $\alpha=-1,0,1$. Moreover, we have

$$
\mid \begin{array}{lll}
G_{-1}(x, 0)=0 & \text { for all } & x \in[0,1] \\
G_{1}(x, e)=0 & \text { for all } & x \in[0,1]
\end{array}
$$

Now, for every $y \in \mathbb{R}^{N}$, we set for each $\alpha=-1,0,1$,

$$
Y_{\alpha}(y)=\operatorname{Proj}_{\left.\right|_{\alpha}}(y) \text {. }
$$

Then we define the extension $G$ of $F$ on $[0,1] \times \mathbb{R}^{N}$ by:

$$
G(x, y)=G_{0}\left(x, Y_{0}(y)\right)+(1-x) G_{-1}\left(x, Y_{-1}(y)\right)+x G_{1}\left(x, Y_{1}(y)\right) .
$$

Clearly, because of (1.99), we have

$$
G(x, y)=F(x, y) \quad \text { for any } \quad(x, y) \in[0,1] \times Q_{0} .
$$

Step 1.1: $G(0, z)=G(1, z+e)$ for any $z \in \mathbb{R}^{N}$.

From the definition of $G$, we have for any $z \in \mathbb{R}^{N}$

$$
\begin{aligned}
& G(1, z)=G_{0}\left(1, Y_{0}(z)\right)+G_{1}\left(1, Y_{1}(z)\right) \\
& G(0, z)=G_{0}\left(0, Y_{0}(z)\right)+G_{-1}\left(0, Y_{-1}(z)\right) .
\end{aligned}
$$

Therefore,

$$
\begin{aligned}
G(1, z+e) & =G_{0}\left(1, Y_{0}(z+e)\right)+G_{1}\left(1, Y_{1}(z+e)\right) \\
& =G_{0}\left(1, Y_{-1}(z)+e\right)+G_{1}\left(1, Y_{0}(z)+e\right) \\
& =F\left(1, Y_{-1}(z)+e\right)+F\left(0, Y_{0}(z)\right)-F(0,0) \\
& =F\left(1, Y_{-1}(z)+e\right)+G_{0}\left(0, Y_{0}(z)\right)-F(1, e) \\
& =G_{0}\left(0, Y_{0}(z)\right)+G_{-1}\left(0, Y_{-1}(z)\right) \\
& =G(0, z)
\end{aligned}
$$

where the second equality follows from Corollary A.2 ii), while the third follows from (1.98) and the fourth follows from the fact that $F(1, e)=F(0,0)$.

Step 1.2: $G(x, y)$ is monotone in $y_{i}$

The result of this step follows from the fact that the orthogonal projection preserves the order (Corollary A.2 $i$ )) and that for any $\alpha=-1,0,1, G_{\alpha}$ is non-decreasing on $[0,1] \times Q_{\alpha}$ w.r.t. $y_{i}$ for all $i \in\{1, \ldots, N\}$. 


\section{Step 1.3: $G$ is globally Lipschitz}

Let $(x, y),(\bar{x}, \bar{y}) \in[0,1] \times \mathbb{R}^{N}$, then

$$
\begin{aligned}
|G(x, y)-G(\bar{x}, \bar{y})| \leq & \left|G_{0}\left(x, Y_{0}(y)\right)-G_{0}\left(\bar{x}, Y_{0}(\bar{y})\right)\right|+|\bar{x}-x| \cdot\left|G_{-1}\left(x, Y_{-1}(y)\right)\right| \\
+ & |1-\bar{x}| \cdot\left|G_{-1}\left(x, Y_{-1}(y)\right)-G_{-1}\left(\bar{x}, Y_{-1}(\bar{y})\right)\right|+|x-\bar{x}| \cdot\left|G_{1}\left(x, Y_{1}(y)\right)\right| \\
+ & |\bar{x}| \cdot\left|G_{1}\left(x, Y_{1}(y)\right)-G_{1}\left(\bar{x}, Y_{1}(\bar{y})\right)\right| .
\end{aligned}
$$

Since for $\alpha=-1,0,1$, the functions $G_{\alpha}$ are Lipschitz continuous and bounded on $[0,1] \times Q_{\alpha}$ and using the fact that the orthogonal projection is 1-Lipschitz, we conclude that

$$
|G(x, y)-G(\bar{x}, \bar{y})| \leq M|(x-\bar{x}, y-\bar{y})|,
$$

where $M=L_{0}+L_{-1}+L_{1}+M_{-1}+M_{1}$, with $L_{\alpha}$ is the Lipschitz constant of $G_{\alpha}, M_{\alpha}$ the $L^{\infty}$ norm of $G_{\alpha}$ for $\alpha=-1,0,1$.

Step 2: extension on $\mathbb{R} \times \mathbb{R}^{N}$

Let $k \in \mathbb{Z}$ and set

$$
\tilde{F}(x+k, y+k e)=G(x, y) \quad \text { for all } \quad(x, y) \in[0,1] \times \mathbb{R}^{N} .
$$

First of all, $\tilde{F}$ is well defined because of Step 1.1. Moreover by construction, we have the periodicity property

$$
\tilde{F}(x+1, y+e)=\tilde{F}(x, y) \quad \text { for any } \quad(x, y) \in \mathbb{R} \times \mathbb{R}^{N} .
$$

In addition, $\tilde{F}$ is Lipschitz continuous, non-decreasing in each $y_{i}$ for $i \in\{1, \ldots, N\}$.

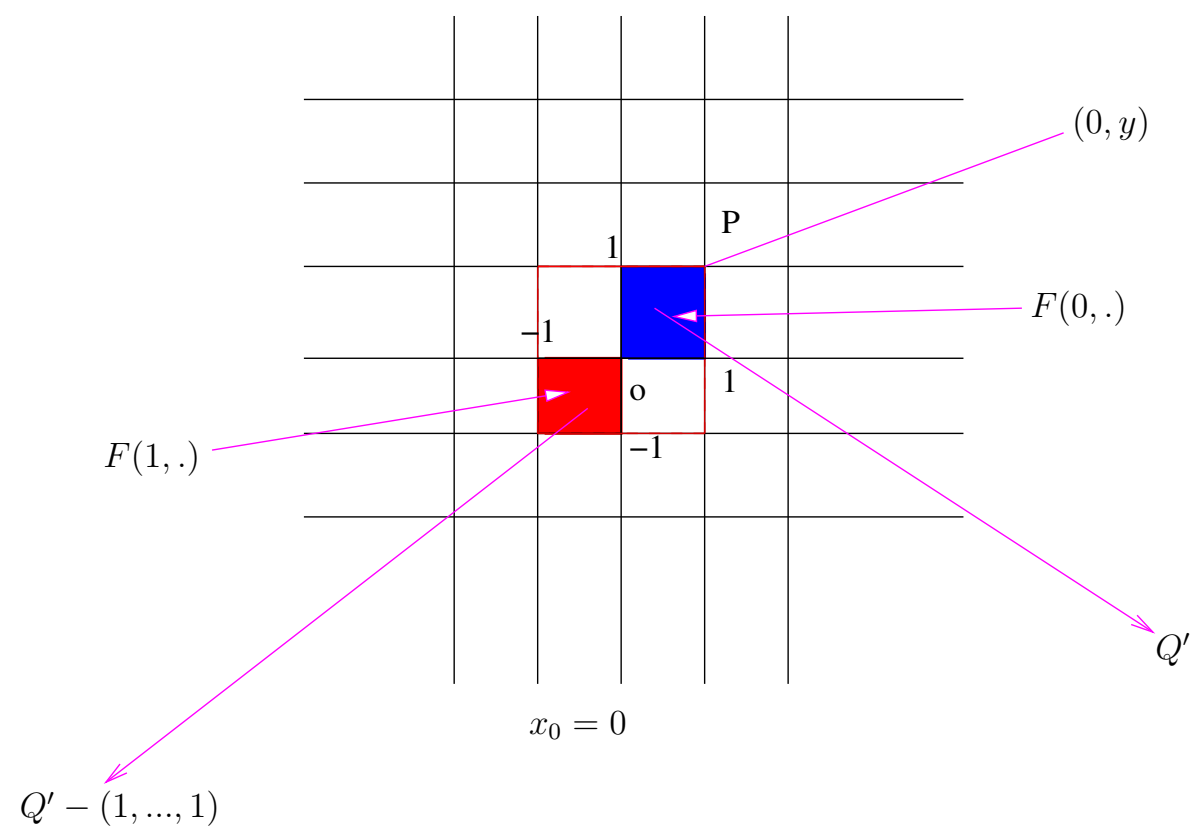

Figure 1: A cut of $\left\{x_{0}=0\right\} \times \mathbb{R}^{N}$ 


\section{Appendix B Proof of miscellaneous properties of mono- tone functions}

Appendix B is dedicated to the proof of some results about monotone functions, namely Lemma 2.10 and Lemma 2.11

We first prove Lemma 2.10.

Proof of Lemma 2.10

Assume for simplicity that $g=0$ on $\stackrel{\circ}{I}$. Suppose to the contrary that there exists a closed interval $I_{0} \subset \stackrel{\circ}{I}, \delta>0$ and a subsequence $x_{n_{j}} \in I_{0}$ with $x_{n_{j}} \rightarrow x_{0} \in I_{0}$ such that

$$
\left|g_{n_{j}}\left(x_{n_{j}}\right)\right| \geq \delta
$$

Assume that $g_{n_{j}}\left(x_{n_{j}}\right) \geq \delta$ (the case $g_{n_{j}}\left(x_{n_{j}}\right) \leq-\delta$ being similar). Let $\varepsilon>0$ and consider a closed interval $I_{\varepsilon}$ such that $I_{0} \subset \subset I_{\varepsilon} \subset \stackrel{\circ}{I}$. Since $g_{n_{j}}(x)$ is non-decreasing in $x$, then

$$
g_{n_{j}}(x) \geq \delta \quad \text { for all } \quad x \in\left(I_{\varepsilon} \backslash I_{0}\right) \cap\left(\left\{x \geq x_{n_{j}}\right\}\right):=I_{+} .
$$

Choose $x_{1} \in I_{+}$such that $g_{n_{j}}\left(x_{1}\right) \rightarrow g\left(x_{1}\right)\left(g_{n}\right.$ converges a.e. on $\left.I_{+}\right)$. Thus

$$
0=g\left(x_{1}\right) \geq \delta>0
$$

a contradiction.

Now, we give the proof of Lemma 2.11. To this end, we recall and prove the following result:

Lemma B.1 (Properties of monotone functions)

Let $\phi: \mathbb{R} \rightarrow \mathbb{R}$ be a non-decreasing function.

i) Countable set of jumps:

The set

$$
\mathcal{S}=\{x \text { such that } \phi \text { is discontinuous at } x\}
$$

is at most countable.

ii) Density of test points:

Let $x_{0} \in \mathbb{R}$, there exists a sequence of functions $\psi_{n}^{+} \in C^{\infty}(\mathbb{R})$ (resp. $\psi_{n}^{-} \in C^{\infty}(\mathbb{R})$ ) and a real sequence $\left(x_{n}^{+}\right)_{n}$ (resp. $\left.\left(x_{n}^{-}\right)_{n}\right)$ such that

$$
x_{n}^{+} \rightarrow x_{0} \quad\left(\text { resp. } \quad x_{n}^{-} \rightarrow x_{0}\right)
$$

and $\phi^{*}-\psi_{n}^{+}$(resp. $\phi_{*}-\psi_{n}^{-}$) attains a local maximum (resp. a local minimum) at $x_{n}^{+}$(resp. at $x_{n}^{-}$) for all $n$.

The meaning of point $i i$ ) is that the set of points where $\phi^{*}$ is tested (in the sense of Definition 2.4 ) from above (resp. $\phi_{*}$ is tested from below) is dense in $\mathbb{R}$.

\section{Proof of Lemma B.1.}

a) Proof of i):

This is classical. 


\section{b) Proof of ii) for $\phi^{*}$ :}

Let $x_{0} \in \mathbb{R}$. We want to prove that there exists $\psi_{n} \in C^{\infty}(\mathbb{R})$ and $x_{n} \rightarrow x_{0}$ such that $\phi^{*}-\psi_{n}$ reaches a local maximum at $x_{n}$. For every $\varepsilon>0$ and for any $b \in \mathbb{R}$, we define the test function

$$
\psi_{n}^{b}=\frac{1}{\varepsilon}\left(x-\left(x_{0}+\frac{1}{n}\right)\right)^{2}+b,
$$

then we set

$$
\beta=\inf \mathcal{E} \quad \text { for } \quad \mathcal{E}=\left\{b \in \mathbb{R}, \quad \psi_{n}^{b}(x) \geq \phi^{*}(x) \quad \forall x \in\left[x_{0}, x_{0}+\frac{2}{n}\right]\right\} .
$$

Indeed, since $\phi^{*}$ is locally bounded (because $\phi$ is a real non-decreasing function) and $\mathcal{E}$ is bounded from below (by definition of $\mathcal{E}$ ), then $\mathcal{E} \neq \emptyset$. From the definition of $\beta$, there always exists $x_{n} \in\left[x_{0}, x_{0}+\frac{2}{n}\right]$ such that

$$
\psi_{n}^{\beta}\left(x_{n}\right)=\phi^{*}\left(x_{n}\right) \quad \text { and } \quad \psi_{n}^{\beta}(x) \geq \phi^{*}(x) \quad \text { on } \quad I=\left[x_{0}, x_{0}+\frac{2}{n}\right] .
$$

We want to show that $x_{n}$ belongs to the interior of $I$ (at least for $\varepsilon$ large enough). We have

$$
\psi_{n}^{\beta}\left(x_{0}\right)=\frac{1}{\varepsilon n^{2}}+\beta>\beta=\psi_{n}^{\beta}\left(x_{0}+\frac{2}{n}\right) \geq \phi^{*}\left(x_{0}+\frac{2}{n}\right) \geq \phi^{*}\left(x_{0}\right),
$$

the last two inequalities are true because of $(2.101)$ and the fact that $\phi^{*}$ is non-decreasing respectively. Assuming

$$
\frac{1}{\varepsilon}>n^{2}\left(\phi^{*}\left(x_{0}+\frac{2}{n}\right)-\phi^{*}\left(x_{0}\right)\right),
$$

we get

$$
\begin{aligned}
\psi_{n}^{\beta}\left(x_{0}+\frac{2}{n}\right) & >\phi^{*}\left(x_{0}+\frac{2}{n}\right)-\phi^{*}\left(x_{0}\right)+\beta \\
& \geq \phi^{*}\left(x_{0}+\frac{2}{n}\right),
\end{aligned}
$$

where the last inequality follows from (2.102). This implies that $\phi^{*}-\psi_{n}^{\beta}$ reaches a local maximum at $x_{n} \in\left(x_{0}, x_{0}+\frac{2}{n}\right)$ and $x_{n} \rightarrow x_{0}$ as $n \rightarrow+\infty$.

c) Proof of ii) for $\phi_{*}$ :

Applying argument b) for $\phi(x)$ replaced by $-\phi(-x)$, we get the result.

\section{Proof of Lemma 2.11.}

We set

$$
\mathcal{T}=\bigcup_{i=0}^{N}\left(\mathcal{S}-\left\{r_{i}\right\}\right)
$$

with $\mathcal{S}$ defined in (2.100). Using Lemma B.1 $i$ ), we get that $\mathcal{T}$ is countable.

Step 1: viscosity sense implies a.e. sense

Assume that $\phi$ is a viscosity solution of (2.27) (see Definition 2.4) and let $x_{0} \in \mathbb{R} \backslash \mathcal{T}$.

By definition, $\phi$ is continuous at $x_{0}+r_{i}$ for all $i=0, \ldots, N$. There exists two sequences of real numbers $\left(x_{n}^{+}\right)_{n}$ and $\left(x_{n}^{-}\right)_{n}$ such that $\phi^{*}$ is tested from above at $x_{n}^{+}$and $\phi_{*}$ is tested from 
below at $x_{n}^{-}$by smooth functions (the sets of such points is dense in $\mathbb{R}$ (by Lemma B.1, $\left.i i\right)$ ), and such that

$$
\lim _{n \rightarrow+\infty} x_{n}^{ \pm}=x_{0}
$$

Moreover, from Definition 2.4, we have

$$
0 \leq F\left(\left(\phi^{*}\left(x_{n}^{+}+r_{i}\right)\right)_{i=0, \ldots, N}\right)
$$

and

$$
0 \geq F\left(\left(\phi_{*}\left(x_{n}^{-}+r_{i}\right)\right)_{i=0, \ldots, N}\right)
$$

Now, using the fact that

$$
\lim _{n \rightarrow+\infty} \phi^{*}\left(x_{n}^{+}+r_{i}\right)=\phi\left(x_{0}+r_{i}\right) \quad \text { for } \quad i=0, \ldots, N
$$

and that $F$ is Lipschitz continuous (see $(\tilde{A})$ ), we pass to the limit $n \rightarrow+\infty$ in $(2.103)$, and we get

$$
\begin{aligned}
0 & \leq \limsup _{n \rightarrow+\infty} F\left(\left(\phi^{*}\left(x_{n}^{+}+r_{i}\right)\right)_{i=0, \ldots, N}\right) \\
& \leq F\left(\left(\phi\left(x_{0}+r_{i}\right)\right)_{i=0, \ldots, N}\right) .
\end{aligned}
$$

Similarly, we show that

$$
\begin{aligned}
0 & \geq \liminf _{n \rightarrow+\infty} F\left(\left(\phi_{*}\left(x_{n}^{-}+r_{i}\right)\right)_{i=0, \ldots, N}\right) \\
& \geq F\left(\left(\phi\left(x_{0}+r_{i}\right)\right)_{i=0, \ldots, N}\right) .
\end{aligned}
$$

Thus

$$
0=F\left(\left(\phi\left(x_{0}+r_{i}\right)\right)_{i=0, \ldots, N}\right),
$$

hence $\phi$ solves equation (2.27) at $x_{0}$. But $x_{0} \in \mathbb{R} \backslash \mathcal{T}$ is arbitrary, thus $\phi$ solves (2.27) pointwisely on $\mathbb{R} \backslash \mathcal{T}$. Since $\mathcal{T}$ is countable, we get that $\phi$ satisfies (2.27) a.e..

\section{Step 2: a.e. sense implies viscosity sense}

Let $x_{0} \in \mathbb{R}$. We want to show that $\phi$ is a viscosity sub-solution at $x_{0}$. Let $\psi \in C^{1}$ such that $\phi \leq \psi$ with equality at $x_{0}$, and we want to prove that

$$
0 \leq F\left(\left(\phi^{*}\left(x_{0}+r_{i}\right)\right)_{i=0, \ldots, N}\right)
$$

Case 1: $x_{0} \notin \mathcal{T}$

If $x_{0} \notin \mathcal{T}$, then $\phi$ is continuous at $x_{0}+r_{i}$ for all $i$. Because $\phi$ solves $(2.27)$ a.e. on $\mathbb{R}$, then there exists a sequence $x_{n} \rightarrow x_{0}$ such that $\phi$ solves (2.27) at $x_{n}$. Hence we have

$$
0=F\left(\left(\phi\left(x_{n}+r_{i}\right)\right)_{i=0, \ldots, N}\right) .
$$

Passing to the limit $n \rightarrow+\infty$, we get

$$
0 \leq F\left(\left(\phi^{*}\left(x_{0}+r_{i}\right)\right)_{i=0, \ldots, N}\right)=F\left(\left(\phi\left(x_{0}+r_{i}\right)\right)_{i=0, \ldots, N}\right) .
$$


Case 2: $x_{0} \in \mathcal{T}$

Now, assume that $x_{0} \in \mathcal{T}$. Since $\mathcal{T}$ is countable, then choose $a_{k}>a_{k+1}>0$ such that $a_{k} \rightarrow 0$ and $x_{0}+a_{k} \notin \mathcal{T}$ for all $k$. Since $x_{0}+a_{k} \notin \mathcal{T}$, then we deduce from Case 1 that

$$
0 \leq F\left(\left(\phi\left(x_{0}+a_{k}+r_{i}\right)\right)_{i=0, \ldots, N}\right) \text {. }
$$

Now letting $a_{k} \rightarrow 0$, we get

$$
\begin{aligned}
0 & \leq \limsup _{a_{k} \rightarrow 0} F\left(\left(\phi\left(x_{0}+a_{k}+r_{i}\right)\right)_{i=0, \ldots, N}\right) \\
& =F\left(\left(\lim _{a_{k} \rightarrow 0} \phi\left(x_{0}+a_{k}+r_{i}\right)\right)_{i=0, \ldots, N}\right) \\
& \leq F\left(\left(\phi^{*}\left(x_{0}+r_{i}\right)\right)_{i=0, \ldots, N}\right) .
\end{aligned}
$$

Here, we use the fact that $\phi^{*}(x)=\lim _{k \rightarrow+\infty} \phi\left(x+a_{k}\right)$ for any $x \in \mathbb{R}$ (because $\phi$ is nondecreasing and $a_{k}>0$ with $\left.a_{k} \rightarrow 0\right)$. Hence $\phi$ is a viscosity sub-solution of (2.27) at $x_{0}$.

Similarly, we show that $\phi$ is a viscosity super-solution at any point, and then $\phi$ is a viscosity solution.

\section{ACKNOWLEDGMENTS}

We wish to thank the unknown referee for his/her advice to improve the presentation of the paper. The first author would like to thank the Lebanese National Council for Scientific Research (CNRS-L) and the Campus France (EGIDE earlier) for supporting him. He also want to thank professor R. Talhouk for his advices. The second author was partially supported by ANR AMAM (ANR 10-JCJC 0106) and ANR IDEE (ANR-2010-0112-01). The last author was also partially supported by the contract ERC ReaDi 321186. Finally, this work was partially supported by ANR HJNet (ANR-12-BS01-0008-01).

\section{References}

[1] L. Ambrosio, N. Gigli, G. Savaré, Gradient flows in metric spaces and in the space of probability measures. Second edition. Lectures in Mathematics ETH Zurich. Birkhauser Verlag, Basel, (2008).

[2] D.G. Aronson, H.F. Weinberger, Nonlinear diffusion in population genetics, combustion, and nerve pulse propagation. Partial differential equations and related topics. Lecture Notes in Math. 446, 5-49. Springer, Berlin, (1975).

[3] G. Barles, Solutions de viscosité des équations de Hamilton-Jacobi. Vol. 17 of Mathématiques \& Applications, Springer-Verlag, Paris, (1994).

[4] P.W. Bates, P.C. Fife, X. Ren, X. Wang, Traveling waves in a convolution model for phase transitions. Arch. Rational Mech. Anal. 138 (2) (1997), 105-136.

[5] P.W. Bates, X. Chen, A. Chmaj, Traveling waves of bistable dynamics on a lattice, SIAM J. Math. Anal. 35 (2) (2003), 520-546. 
[6] H. Berestycki, F. Hamel, Front propagation in periodic excitable media. Comm. Pure Appl. Math. 55 (8) (2002), 949-1032.

[7] H. Berestycki, B. Nicolaenko, B. Scheurer, Traveling wave solutions to combustion models and their singular limits. SIAM J. Math. Anal. 16 (6) (1985), 1207-1242.

[8] O.M. Braun, Y.S. Kivshar, The Frenkel-Kontorova model, Concepts, Methods and Applications. Springer-Verlag, (2004).

[9] A. Carpio, S.J. Chapman, S. Hastings, J.B. McLeod, Wave solutions for a discrete reaction-diffusion equation. European J. Appl. Math. 11 (4) (2000), 399-412.

[10] X. Chen, J.-S. Guo, C.-C. Wu, Traveling waves in discrete periodic media for bistable dynamics. Arch. Ration. Mech. Anal. 189 (2) (2008), 189-236.

[11] X. CHen, Existence, uniqueness, and asymptotic stability of traveling waves in nonlocal evolution equations. Adv. Differential Equations 2 (1) (1997), 125-160.

[12] S.-N Chow, J. Mallet-Paret, W. Shen, Traveling waves in lattice dynamical systems. J. Differential Equations 149 (2) (1998), 248-291.

[13] A. De Masi, T. Gobron, E. Presutti, Travelling fronts in non-local evolution equations. Arch. Rational Mech. Anal. 132 (2) (1995), 143-205.

[14] C.E. Elmer, E.S. VAN Vleck, A variant of Newton's method for the computation of traveling waves of bistable differential-difference equations. J. Dynam. Differential Equations 14 (3) (2002), 493-517.

[15] P.C. FIFE, J.B. MCLEOD, The approach of solutions of nonlinear diffusion equations to travelling front solutions. Arch. Ration. Mech. Anal. 65 (4) (1977), 335-361.

[16] R.A. Fisher, The advance of advantageous genes. Ann. Eugenics 7 (1937), 335-369.

[17] J.-S. Guo, Y.-C. Lin, Traveling wave solution for a lattice dynamical system with convolution type nonlinearity. Discrete Contin. Dyn. Syst. 32 (1) (2012), 101124.

[18] D. Hankerson, B. Zinner, Wavefronts for a cooperative tridiagonal system of differential equations. J. Dynam. Differential Equations 5 (2) (1993), 359-373.

[19] J. I. KAnEL', Certain problems on equations in the theory of burning. Dokl. Akad. Nauk SSSR 136 277-280 (Russian); translated as Soviet Math. Dokl. 2 (1961), 48-51.

[20] A.N. Kolmogorov, I.G. Petrovsky, N.S. Piskunov, Etude de l'équation de la diffusion avec croissance de la quantité de matière et son application à un problème biologique, Bull. Université d'Etat à Moscou, Ser. Int. Sect. A. 1. (1937), 1-25.

[21] J. MALlet-PARET, The Fredholm alternative for functional differential equation of mixed type. J. Dynam. Differential Equations 11 (1) (1999), 1-47.

[22] J. MAllet-PARET, The global structure of traveling waves in spatially discrete dynamical systems. J. Dynam. Differential Equations 11 (1) (1999), 49-127. 
[23] N. Forcadel, C. Imbert, R. Monneau, Homogenization of fully overdamped Frenkel-Kontorova models. J. Differential Equations 246 (3) (2009), 1057-1097.

[24] J. Wu, X. Zou, Asymptotic and periodic boundary value problems of mixed FDEs and wave solutions of lattice differential equations, J. Differential Equations 135 (2) (1997), 315-357.

[25] B. Zinner, Existence of traveling wavefront solutions for the discrete Nagumo equation. J. Differential Equations 96 (1) (1992), 1-27.

[26] B. Zinner, G. Harris, W. Hudson Traveling wavefronts for the discrete Fisher's equation, J. Differential Equations 105 (1) (1993), 46-62. 